\title{
Advances in Clinical Cardiology 2018: A Summary of Key Clinical Trials
}

\author{
Katie Linden · Conor McQuillan · Paul Brennan · Ian B. A. Menown
}

Received: March 29, 2019 / Published online: May 7, 2019

(C) The Author(s) 2019

\section{ABSTRACT}

Introduction: Many important clinical trials in cardiology were published or presented at major international meetings throughout 2018. This paper aims to offer a concise overview of these significant advances and to put them into clinical context.

Methods: Trials presented at the major international cardiology meetings during 2018 were reviewed including The American College of Cardiology, EuroPCR, The European Society of Cardiology, PCR London Valves, Transcatheter Cardiovascular Therapeutics, and the American Heart Association. In addition to this a literature search identified several other publications eligible for inclusion based on their relevance to clinical cardiology, their potential impact on clinical practice and on future guidelines.

Results: A total of 78 trials met the inclusion criteria. New interventional and structural data include trials examining novel stent designs $\left(\right.$ Biofreedom $\left.^{\mathrm{TM}}, \mathrm{COMBO}\right)$, use of drug-coated

Enhanced Digital Features To view enhanced digital features for this article go to https://doi.org/10.6084/ m9.figshare.7993331.

K. Linden $(\bowtie) \cdot$ C. McQuillan · P. Brennan ·

I. B. A. Menown

Craigavon Cardiac Centre, SHSCT, Craigavon,

Northern Ireland, UK

e-mail: kmslinden@gmail.com balloons in patients with high bleeding risk, intervention in stable coronary artery disease, revascularisation strategy in ST elevation myocardial infarction, transcatheter aortic valve replacement in low-risk patients, and percutaneous mitral or tricuspid valve interventions. Preventative cardiology data included the use of sodium glucose cotransporter-2 inhibitors (empagliflozin, dapagliflozin, canagliflozin), proprotein convertase subtilisinkexin type 9 (PCSK9) inhibitors (alirocumab) and approaches of hypertension management. Antiplatelet data included trials evaluating both the optimal length of course and combination of antiplatelet agents. Heart failure data included trials of sacubitril-valsartan during acute hospital admission and the management of chemotherapy-induced cardiotoxicity. Electrophysiology data included trials examining atrial fibrillation ablation, wearable cardiac defibrillators (LifeVest) and His-bundle pacing.

Conclusion: This article presents key clinical trials completed during 2018 and should be valuable to both cardiology clinicians and researchers.

Keywords: Acute coronary syndrome; Anticoagulation; Atrial fibrillation; Cardiology; Coronary revascularization; Heart failure; Lipids; Mitral Clip; Myocardial infarction; Transcatheter aortic valve implantation 


\section{INTRODUCTION}

A multitude of clinical trials were published and presented during 2018, with the potential to impact on cardiology clinical practice and influence future guidelines, including presentations at The American College of Cardiology (ACC), EuroPCR, European Society of Cardiology (ESC), PCR London Valves, Transcatheter Cardiovascular Therapeutics (TCT) and the American Heart Association (AHA). In this paper we review key studies within the categories of interventional cardiology, structural cardiology, cardiovascular (CV) prevention, acute coronary syndrome (ACS), electrophysiology and heart failure.

\section{METHODS}

The results of clinical trials presented at major international cardiology meetings in 2018 were reviewed. In addition to this a literature search of PubMed, Medline, Cochrane library and Embase was completed including the terms "acute coronary syndrome", "atrial fibrillation", "coronary prevention", "electrophysiology", "heart failure" and "interventional cardiology". Trials were assessed for relevance to clinical cardiology and the potential impact on clinical practice and future guidelines. This article is based on previously completed work and does not involve any new studies of human or animal subjects performed by any of the authors.

\section{ADVANCES IN INTERVENTIONAL CARDIOLOGY}

The COMBO stent (OrbusNeich Medical BV) is a $100-\mu \mathrm{m}$ strut stainless steel stent with a sirolimus-eluting biodegradable polymer and a pro-healing layer of immobilized anti-CD34 antibodies, designed to attract circulating endothelial progenitor cells that can rapidly differentiate into normal endothelium. Previous studies have suggested non-inferiority vs. Xience albeit with wide confidence intervals to define this [1]. A new pooled analysis from the large MASCOT $(n=2614)$ and REMEDEE registries $(n=1000)$ reported low rates of cardiac death $(1.6 \%)$, target vessel myocardial infarction (MI) (1.2\%) and target lesion revascularisation (TLR) $(2.2 \%)$ at 1-year follow-up in all-comers populations [2]. In addition, definite stent thrombosis was low at $0.5 \%$ suggesting good safety associated with the COMBO prohealing coating.

The risk of late and very late stent thrombosis remains a key concern when considering optimal stent choice. The ISAR-TEST4 trial suggested a safety advantage with newer-generation drug-eluting stents (DES). A total of 2603 patients were assigned to biodegradable polymer-based sirolimus-eluting stents (BP-SES; Yukon Choice PC) $(n=1299)$ vs. second-generation permanent polymer-based everolimuseluting stents (PP-EES; Xience) $(n=652)$ vs. first-generation permanent polymer-based sirolimus-eluting stents (PP-SES; Cypher) $(n=652)$. At 10 -year follow up, the incidence of major adverse cardiac events (MACE) was significantly lower with newer-generation stents (BP-SES $47.7 \%$ vs. PP-EES $46.0 \%$ vs. PP-SES $54.9 \%, P=0.003$ ) as was mortality (BP-SES $31.8 \%$ vs. PP-EES $30.3 \%$ vs. PP-SES $37.2 \%$, $P=0.02$ ) and definite stent thrombosis (BP-SES $1.1 \%$ vs. PP-EES $0.8 \%$ vs. PP-SES $2.4 \%, P=0.03$ ) [3]. Although this post hoc analysis was limited by a median follow-up of 5.9 years, the lower event rates with new-generation DES was marked. Of note, among the new DES, there was no clear difference observed between those with biodegradable or permanent polymers suggesting improved polymer biocompatibility overall. However, current meta-analyses comparing biodegradable versus newer permanent polymer stents remain limited by relatively short followup [4].

Superiority of the polymer-free Biolimus A9coated stent Biofreedom $^{\mathrm{TM}}$ (BA9-DCS) vs. bare metal stent (BMS) in high bleeding risk (HBR) patients receiving just 1 month of dual antiplatelet therapy (DAPT) [5]. Given the short course of DAPT, it was important to assess the strategy in patients with more complex disease. Two-year follow-up of the complex PCI (including bifurcation lesions and chronic total occlusions, CTOs) subset $(n=667)$ reported significant reduction in clinically driven TLR 
much lower with BA9-DCS vs. BMS (10.8\% vs. $18.1 \%, P<0.005)$. Given the superiority of DES vs. BMS for routine PCI and superiority of BA9DCS vs. BMS for HBR patients, the need for use of BMS in contemporary PCI is now minimal [6].

Polymer-free stent technology was also compared with DES in the ReCre8 trial, which evaluated the efficacy of the polymer-free amphilimus-eluting stent (PF-AES) vs. latestgeneration permanent-polymer zotarolimuseluting stents (PP-ZES) (Resolute Integrity, Medtronic Vascular) in a multicentre trial of 1491 all-comers patients. DAPT duration was 12 months for ACS patients and 1 month for troponin-negative patients [7]. At 12 months, there was no difference in cardiac death $(1.3 \%$ vs. $1.3 \%, P=1.0)$, target vessel $\mathrm{MI}(2.4 \%$ vs. $2.3 \%, P=0.87)$ or TLR $(2.9 \%$ vs. $2.6 \%, P=0.75)$ indicating that despite the absence of a permanent polymer and thus shorter drug elution, the PF-AES stent was non-inferior to PP-ZES.

Previous trials of bioresorbable vascular scaffolds (BVS) have been disappointing, associated with increased late target lesion failure (TLF), target vessel MI and ST, especially in small vessels $(<2.25 \mathrm{~mm}$ diameter) [8]. Clinical use of the Abbott BVS has been suspended [9], but clinical trials have continued follow-up [10].

The Intracoronary Scaffold Assessment a Randomised evaluation of Absorb in Myocardial Infarction (ISAR-Absorb MI) trial randomised 262 patients with ST-elevation myocardial infarction (STEMI) and non-STEMI (NSTEMI) to PCI with everolimus-eluting BVS (Absorb; Abbott Vascular) (Fig. 1) vs. everolimus-eluting DES (Xience; Abbott International). There was no significant difference in the primary endpoint of percentage diameter stenosis; at 6-month angiography rates of TLR, CV death and ST were comparable [11]. However, given that late target lesion failure was seen in prior studies, on-going follow-up is awaited.

Given concerns that disappointing results in prior BVS studies may have been related, at least in part, to suboptimal implantation technique, ABSORB IV randomised patients with stable coronary artery disease (CAD) or ACS to Absorb BVS $(n=1296) \quad$ implanted with

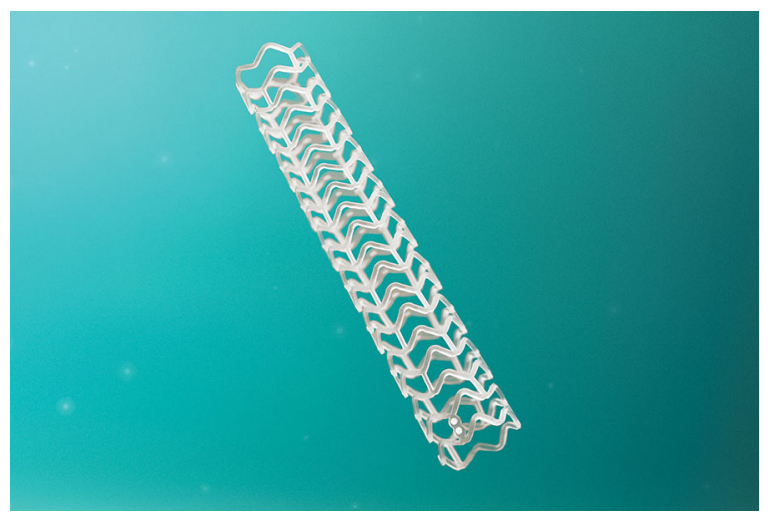

Fig. 1 Absorb bioresorbable vascular scaffold system. Image provided courtesy of Abbott Vascular. (C)2019/ 2020 Abbott. All Rights Reserved

optimised operator technique vs. Xience DES $(n=1308)$. A small numerical excess in events was still noted with BVS vs. Xience, although statistical criteria for non-inferiority were satisfied both at 30 -day TLF $(5.0 \%$ vs. $3.7 \%$; $\left.p_{\text {non-inferiority }}=0.0244\right)$ and 1 -year TLF $(7.8 \%$ vs. $\left.6.4 \%, p_{\text {non-inferiority }}=0.0006\right)[12]$.

In the COMPARE-ABSORB trial, patients with increased risk of restenosis (diabetes, multivessel disease, lesion length $>28 \mathrm{~mm}$, small vessels between 2.25 and $2.75 \mathrm{~mm}$ and bifurcation with single device strategy) were randomised to Absorb BVS $(n=848)$ vs. Xience DES $(n=822)$. The study was terminated early by the Data Safety and Monitoring Board following safety concerns arising from ABSORB II and III trials. At 1-year follow-up of patients already randomised, BVS was non-inferior to Xience for TLF $\left(5.1 \%\right.$ vs. $4.2 \%$, $\left.p_{\text {non-inferiority }}<0.001\right)$, but was associated with excess target vessel MI (4.0\% vs. $2.1 \%, P=0.02$ ) and device thrombosis (2.0\% vs. $0.6 \%, P=0.01)$ [13].

Several other absorbable stents including MeRes100 (a thin strut, second-generation sirolimus-eluting BVS) and DREAMS 2G (a drugeluting bioabsorbable magnesium-based scaffold) have reported promising early results. However, definitive trials of these newer devices vs. DES are still awaited [14, 15].

Drug-eluting balloons (DEB) (also known as drug-coated balloons) are recommended by the ESC guidelines for the treatment of in-stent restenosis (ISR) (level of evidence IA) [10] but 
their role in de novo lesions is less well established, although small studies such as Valentines II have suggested a potential role [16].

The DEBUT trial (Drug-Eluting Balloon in stable and Unstable angina: a randomized controlled non-inferiority Trial) compared PCI with DEB vs. BMS in patients with de novo CAD and at HBR [17]. The trial was stopped early because of excess events in the BMS group. At 9 months, use of DEB was associated with lower MACE (1.9\% vs. $12.4 \%, P<0.001$ for noninferiority; $P=0.004$ for superiority) and a trend to lower TLR ( $0 \%$ vs. $4.8 \% ; P<0.001$ for noninferiority; $P=0.06$ for superiority). This supports consideration of DEB-only PCI in patients at HBR.

DEBs may be useful in smaller-diameter vessels where ISR risks are higher. The BASKETSMALL 2 trial randomised 758 patients (1:1) with small-calibre coronary arteries $(<3 \mathrm{~mm})$ and an indication for PCI to DEB vs. DES [18]. After 12 months, cumulative MACE was similar in both groups [7.5\% DEB vs. $7.3 \%$ DES, (HR) 0.97 (95\% CI 0.58-1.64), $P=0.9180$ ] supporting DEB as a reasonable alternative to DES in patients with small coronary arteries. It is worth noting that this study may have been limited by the inclusion of coronary arteries of up to $3 \mathrm{~mm}$ calibre when usually small calibre is defined as $<2.5 \mathrm{~mm}$.

As new techniques and technologies become available for CTO revascularisation, the longterm outcomes from dissection re-entry and subintimal stenting procedures are of importance. The Consistent CTO (CONventional antegrade vs. Sub-Intimal Synergy sTENTing in Chronic Total Occlusions) trial reported 1-year outcomes from 210 patients (average Japanese CTO score 2.4) undergoing CTO intervention using antegrade (antegrade wire escalation 34\%, antegrade dissection re-entry 18\%) and retrograde (retrograde wire escalation 18\%, retrograde dissection re-entry $30 \%$ ) techniques. In total, $90 \%$ of CTOs were successfully opened (210/231 patients), with a mean lesion length of $29.1 \mathrm{~mm}$ and procedural time $122 \mathrm{~min}$ [19]. At 1 year, MACE (all-cause death, MI and TVR) was $10 \%$ with low target vessel failure rate $5.2 \%$ and stent thrombosis $1.4 \%$. Patients reported substantial gains in QOL scores with low MACE rate despite complex disease and revascularisation techniques.

The optimal place for PCI in patients with stable CAD continues to be debated. Five-year follow-up from the FAME 2 trial (PCI guided by fractional flow reserve (FFR) in patients with stable coronary artery disease) reported favourable long-term outcomes in patients without haemodynamically significant stenosis being treated with medical therapy alone. Of the 888 patients randomised (447 PCI vs. 441 medical therapy alone) [20], the primary endpoint (composite of death, MI and urgent revascularisation) reached statistical significance $(13.9 \%$ vs. $27.0 \%, P<0.001$ ) but was driven by urgent revascularisation in the medical therapy group (6.3\% vs. $21.1 \%)$ with no significant difference in death or MI at 5 years.

The ORBITA trial (Percutaneous Coronary Intervention in Stable Angina) discussed in detail previously [8] showed only a small but non-significant improvement in treadmill exercise time for PCI vs. sham procedure. However, pre-randomisation intracoronary physiology assessment with FFR or instantaneous wave-free ratio (iFR) was available in 196 patients (103 PCI vs. 93 sham procedure) with mean FFR 0.69 and iFR 0.76 [21]. In a subsequent analysis the blinded effect of PCI was noted to be more clearly seen by stress echocardiography score and freedom from angina than change in treadmill exercise time. Moreover, the lower the FFR or iFR at baseline, the greater the magnitude of stress echocardiographic improvement caused by PCI.

Multivessel CAD is observed in 50\% of patients presenting with STEMI with treatment strategy of the non-infarct-related artery remaining a point of contention between interventional cardiologists. The safety and efficacy of FFR-guided multivessel angioplasty in STEMI patients has been established in the COMPARE-ACUTE trial [22]. Follow-up to 2 years confirmed the initial findings with no change in MI, death or stroke rate but a significantly higher rate of subsequent revascularisation was observed in those where infarct-related artery-only PCI was undertaken [HR 0.41, 95\% CI (0.29-0.59), $P<0.0001]$ [23]. Further studies are required to establish the optimal timing (i.e. 
in-hospital vs. early elective timing) for revascularization of non-infarct related CAD.

Cardiogenic shock complicating acute MI carries a significant mortality rate. The CULPRIT SHOCK trial demonstrated a reduction in 30-day mortality in patients treated with culprit-lesion-only PCI vs. complete revascularisation during the acute presentation $(45.9 \%$ vs. $55.4 \%, P=0.01$ ) leading to a change in the most recent ESC revascularisation guidelines, downgrading complete revascularisation in cardiogenic shock to level III $[10,24]$. The 12-month follow-up from CULPRIT SHOCK has seen a sustained benefit with culprit-lesion-only PCI with reduced mortality $(50.0 \%$ vs. $56.9 \%)$ cementing culprit-lesion-only PCI as the preferred default strategy in patients with cardiogenic shock [25].

Current guidelines recommend an invasive strategy for patients with NSTEMI $<72 \mathrm{~h}$; however, the optimal timing of assessment is not well defined [10]. The VERDICT trial randomised 2147 patients with NSTE-ACS to early invasive coronary angiography and revascularisation ( $<12 \mathrm{~h}$, median $4.7 \mathrm{~h})$ vs. standard care (48-72 h, median $61.6 \mathrm{~h}$ ). Over a median follow-up of 4.3 years the primary endpoint (death, non-fatal MI, hospital admission with ischaemia or heart failure [HF]) was not significantly reduced overall by an early invasive strategy (27.5\% vs. $29.5 \%$ ) [26], although benefit with an early strategy was suggested in patients with elevated GRACE risk score (Global Registry of Acute Coronary Events) $>140$ (HR $0.81 ; 95 \%$ CI 0.67 1.01) in keeping with current PCI guidelines. Findings from the ongoing RAPID-CTCA trial (The Rapid Assessment of Potential Ischaemic Heart Disease with CTCA) may help define the role of CTCA for early assessment of patients with suspected or confirmed ACS [27].

Measurement of FFR in angiographically moderate lesions not only changes revascularisation strategy but leads to better outcome for patients as established in the FAME trials. However, pressure wire assessment takes additional time and resources and is occasionally associated with complications including dissection and distal wire perforation. The FASTFFR (FFR angio Accuracy vs. Standard FFR) trial compared non-invasive (FFR $\mathrm{Fngio}_{\text {an }}$ software analysis of two angiographic views) versus pressure wire FFR assessment of 319 lesions in 301 patients. The diagnostic accuracy of $\mathrm{FFR}_{\text {angio }}$ was high overall (92\%) and remained good (87\%) when considering grey zone FFR values between 0.75 and 0.85 [28]. The improved time efficiency of $\mathrm{FFR}_{\text {angio }}$ may encourage operators to make more routine use of physiology during coronary lesion assessment.

Patients with symptoms of ischaemia but no obstructive coronary artery disease (INOCA) may be difficult to diagnose and treat. In the CorMicA trial, 151 patients with angina but no obstructive disease at angiography were randomised to additional interventional diagnostic (ID) assessment (coronary flow reserve, index of microcirculatory resistance, fractional flow reserve, acetylcholine testing) then stratified medical therapy guided by ID results versus a sham assessment and standard care (ID results undisclosed). Those receiving additional IDguided medical therapy had significant improvements in Seattle Angina Questionnaire, mean quality-of-life score and visual analogue score at 6 months, although there was no significant difference in MACE [29].

Intracoronary imaging is increasingly being utilised to optimise coronary intervention. In the Lipid-Rich Plaque (LRP) study [30], 1552 patients undergoing de novo culprit lesion PCI disease also received imaging with a combined (NIRS) near-infrared spectroscopy-IVUS (intravascular ultrasound) catheter. The system displays a "chemogram" in which clear coronary arteries appear red, lipid-rich plaque appears yellow and calculates the maximum lipid core burden in a 4-mm segment (maxLCBI4mm).

NIRS-IVUS was performed on at least two non-culprit arteries. All patients with at least one maxLCBI $4 \mathrm{~mm}$ segment $\geq 250$ and half of patients (randomly selected) with all maxLCBI4mm segments $<250$ were followed for 2 years. On adjusted patient-level analysis $(n=1271)$, each 100-unit increase in maxLC$\mathrm{BI} 4 \mathrm{~mm}$ was associated with an $18 \%$ higher risk of a non-culprit MACE event. Patients with $\operatorname{maxLCBI} 4 \mathrm{~mm} \geq 400$ vs. $<400$ had double the incidence of MACE $(12.6 \%$ vs. $6.3 \%$, 
respectively). NIRS-IVUS thus appears a practical invasive method to identify vulnerable plaques. Prospective studies are now required to assess if medical or interventional treatment of these plaques improves clinical outcomes.

Radial access has become the default for invasive coronary angiography. Anticoagulation strategy, however, varies between operators. The MATRIX (Minimizing Adverse Haemorrhagic Events by Transradial Access Site and Systemic Implementation of Angiox) program compares the effectiveness of radial vs. femoral access, and bivalirudin vs. unfractionated heparin in patients with ACS undergoing invasive management [31]. At 1 year, use of radial vs. femoral access was associated with a small, non-significant reduction in 1-year MACE $(14.2 \%$ vs. $15.7 \% ; P=0.052)$ and a significant reduction in net adverse clinical events, driven by fewer bleeding complications (15.2\% vs. $17.2 \% ; P=0.01$ ). However, use of bivalirudin vs. heparin was not associated with any significant reduction in MACE, net clinical events, ST or urgent TLR.

\section{ADVANCES IN STRUCTURAL CARDIOLOGY}

Improving imaging modalities, procedural advancements and outcomes from global studies have led to progressive expansion in the application of structural heart interventions.

Transcatheter aortic valve implantation (TAVI) has evolved markedly since Cribier's first "in-human" TAVI in Rouen in 2002 [32]. TAVI for severe symptomatic aortic stenosis in patients at "high or intermediate" operative risk is well established in global guidelines [33-35]. The past year saw several groups report outcomes of TAVI in patients at low operative risk. The NOTION (Nordic Aortic Valve Intervention) investigators reported 5-year outcomes for patients with severe aortic stenosis at low operative risk randomised to TAVI $(n=139)$ vs. surgical aortic valve replacement [SAVR $(n=135)$ ] [36]. At 5 years, TAVI showed no difference in the primary endpoint of all-cause mortality, MI or stroke $(31.5 \%$ vs. $35.2 \%$, $P=0.51)$. TAVI was associated with increased need for new permanent pacemaker (PPM) implantation $(41.8 \%$ vs. $8.4 \%, P<0.001)$. There was an associated increase in mortality for new PPM TAVI patients vs. no new PPM SAVR patients $(38.2 \%$ vs. $21.7 \%, P=0.07)$. At least moderate aortic regurgitation (AR) was only seen in the TAVI group at 5 years $(8.2 \%$, $P<0.001$ ). Long-term outcomes ( $\geq 10$ years) will be of key interest, in particular with regards to the durability of TAVI in this low-risk patient group.

The Low Risk TAVR (LRT) investigators reported a prospective, multicentre, unblinded registry of TAVI in low-risk (STS-PROM $<4 \%$ ) patients $(n=200)$ compared with historical outcomes from the Society of Thoracic Surgeons (STS) database of patients that underwent isolated SAVR $(n=719)$ at enrolling centres from January 2013 to December 2017 [37]. There was no significant difference in adjusted mean age or STS-PROM score in TAVI vs. SAVR groups. The primary endpoint of all-cause mortality was $0 \%$ with TAVI group vs. $1.7 \%$ with SAVR $(P=0.079)$. By 30 days, TAVI was associated with $0 \%$ stroke, only $0.5 \%$ mild or greater paravalvular leak (PVL) and a modest 5\% PPM implantation rate. Subclinical leaflet thrombosis (SLT), assessed by multi-detector CT (MDCT, $n=192$ ) and transoesophagael echo (TEE, $n=2$ ), was observed in $14 \%$ of the TAVI group, similar to results seen in the SAVORY (Subclinical Aortic Valve Bioprosthesis Thrombosis Assessed with Four-Dimensional Computed Tomography) registry [38].

The GARY (German Aortic Valve Registry) investigators also reported outcomes from all patients with a low operative risk, STSPROM $<4 \%$, that underwent isolated TAVI $(n=6062)$ or isolated SAVR $(n=14,487)$ [39]. Of note, overall baseline characteristics of the two groups significantly differed with the TAVI group being older (79 vs. 67 years, $P<0.0001$ ) and of higher predicted perioperative risk (STSPROM $2.9 \%$ vs. $1.79 \%, P<0.0001$ ), but after adjusting for this in a weighted propensity scoring model, 30-day mortality was significantly less with TAVI $(1.9 \%$ vs. $2.9 \%, P=0.014)$ and 1 -year mortality was similar (10\% vs. $8.8 \%$, $P=0.158)$. 
The NOTION, LRT and GARY outcomes demonstrate the feasibility of TAVI in low-risk patient groups but longer-term follow-up, particularly regarding valve durability, is required before TAVI can be routinely considered in this patient group. Results of PARTNER 3 and Medtronic Evolut Transcatheter Aortic Valve Replacement in Low Risk Patients trials, expected in 2019, will add to the evidence base.

The FRANCE-2 (French Aortic National Corevalve and Edwards) registry analysed 4201 high-risk patients undergoing TAVI at 34 French centres between January 2010 and January 2012 [40]. At 5 years, all-cause mortality was $60.8 \%$. Of 459 patients with echo assessment at 5 years, haemodynamic structural valve deterioration (SVD), as defined by the European Association of Percutaneous Interventions (EAPCI), was observed as moderate in 13.3\% and severe in $2.5 \%$, in keeping with prior data and suggesting acceptable durability at 5 years $[41,42]$.

There were several technological advancements in TAVI over the past year. Firstly, Edwards Lifesciences (Irvine, CA, USA) received CE approval for the CENTERA transcatheter heart valve (THV) [43]. The CENTERA THV is a novel self-expanding nitinol valve, which has a short frame height and unique contoured shape. It is delivered using a motorised, steerable delivery system that allows for more precise positioning, stable valve deployment and the ability to recapture the valve up to $80 \%$ of the deployment (Fig. 2).

The CENTERA-EU trial has now reported their outcomes at 1 year for patients with severe aortic stenosis $(n=198)$. The primary endpoint of all-cause mortality at 30 days was $1 \%$. At 1 year, all-cause mortality was 9.1\%. New PPM implantation rates at 30 days were low at less than $5 \%$ and the rate of disabling stroke at 1 year was $4.1 \%$. THV haemodynamics were favourable at 1 year with a mean aortic THV gradient of $8.1 \mathrm{mmHg}$ and no observed cases of moderate or severe AR [44]. However, we await further outcomes at 2, 5 and 10 years, which will be key in assessing the longer-term durability of this novel device.

Early PORTICO 1 outcomes for the Abbott self-expanding Portico THV in high-risk patients with symptomatic aortic stenosis ( $n=941$, mean STS-PROM 5.8\%) [45] reported procedural success of $96 \%$, a primary endpoint of all-cause mortality at 1 year of $12.1 \%$ and stroke rate of $2.2 \%$ cases (most of which occurred within 30 days). Haemodynamic outcomes were good at 1 year with a mean aortic THV gradient of $8.7 \mathrm{mmHg}$. Moderate PVL was seen in $2.6 \%$ at 1 year but severe PVL was not observed. PPM implantation rate at 1 year was high at $21.3 \%$, not dissimilar to the $19.7 \%$ seen in the FORWARD (Evolut R) study but higher than that in CENTERA-EU $[44,46]$.
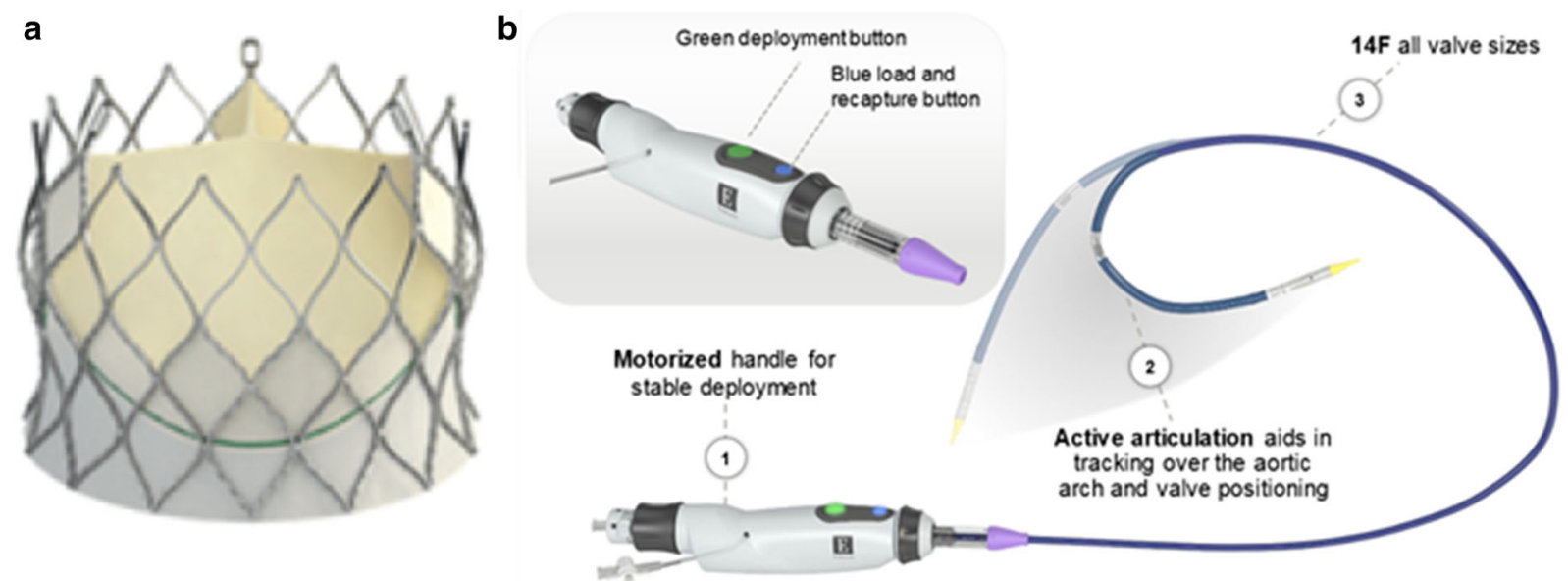

Fig. 2 a CENTERA transcatheter heart valve. b CENTERA delivery system. Images permitted and courtesy of Edwards Lifesciences (Irvine, CA, USA) 
The SOLVE-TAVI study randomised 447 patients with severe aortic stenosis to the selfexpanding Evolut R (Medtronic) THV vs. balloon-expandable Sapien 3 (Edwards Lifesciences) THV. There was no difference in the combined primary endpoint of all-cause mortality, stroke, moderate/severe valve regurgitation and PPM implantation at 30 days $(27.2 \%$ vs. $26.1 \%, p$ for equivalence $=0.02$ ). Higher stroke rate was seen with the Sapien $3(0.5 \%$ vs. $4.7 \% ; P=0.01$ ) and PPM implantation was high in both arms $(22.9 \%$ vs. $19 \% ; P=$ ns) [47]. The similar primary outcome rates support THV choice being made by an experienced heart team and tailored to individual anatomical characteristics.

Valve-in-Valve TAVI (ViV) for aortic bioprosthetic valve (BPV) failure has a IIaC recommendation in ESC Valvular Heart Disease Guidelines [32] but a potential pitfall is prosthesis-patient mismatch especially in smaller $(\leq 21 \mathrm{~mm})$ BPVs due to limitation of THV maximum effective orifice area (EOA) by the dimensions of the original BPV frame. Use of high-pressure non-compliant balloon inflation to cause bioprosthetic valve fracture (BVF) either after or before ViV TAVI was studied in 75 patients at 21 centres with small BPVs (median internal diameter of $18.5 \mathrm{~mm}$ ). Final mean transvalvular gradient was lower when BVF was performed after vs. before VIV TAVI $(8.1 \pm 4.8 \mathrm{mmHg} \quad$ vs. $\quad 16.9 \pm 10.1 \mathrm{mmHg}$; $P<0.001)$ [48].

Periprocedural stroke rates, occurring after TAVI, have diminished with advancing techniques and valve design but remain around 4.4\% [49]. The SENTINEL study reported that patients randomised to the Setinel Cerebral Protection System $(n=280)$ (Claret Medical) vs. unprotected TAVI had an adjusted $42 \%$ reduction in new cerebral lesion volume and a 63\% reduction in clinical stroke within $72 \mathrm{~h}$ of TAVI [50]. Further registry data also reported lower stroke incidence in SENTINEL protected vs. unprotected patients in the first week after TAVI (1.4\% in 291 patients vs. $6.3 \%$ in 128 unprotected patients) [51]. However ongoing study is needed to deem whether cerebral protection should be used routinely in all patients undergoing TAVI or restricted to those patients at the highest stroke risk.

SLT, seen on MDCT, can progress to haemodynamic consequence, but the benefit of oral anticoagulation (OAC) has been unclear. Analysis of 11,469 high-risk patients (mean age 82.8 years, logistic EuroScore 17.8\%) undergoing TAVI between 2013 and 201 from the FRANCE-TAVI registry [52] reported that use of OAC on discharge was associated with significantly less SVD in the 2555 patients with at least two echo evaluations (adjusted OR 0.54, $P=0.05)$, as was a non-femoral approach. Despite this, use of OAC on discharge was associated with an $18 \%$ relative risk increase of death at 3 years, although this may have been in part related to high baseline comorbidity in patients on OAC (including rates of chronic renal failure, logistic EuroScore and NYHA status).

Concerns regarding OAC were also noted in GALILEO trial which randomised patients post TAVI to an OAC arm (10 $\mathrm{mg}$ rivaroxaban OD + aspirin 75-100 $\mathrm{mg}$ for 90 days followed by rivaroxaban alone) vs. antiplatelet arm (clopidogrel $75 \mathrm{~g}+$ aspirin $75-100 \mathrm{mg}$ ). The study was stopped early after 1644 patients were randomised and the OAC arm was associated with a higher rate of all-cause mortality or first thromboembolic event $(11.4 \%$ vs. $8.8 \%)$ and bleeding $(4.2 \%$ vs. 2.4\%) [53].

Early discharge after TAVI is desirable, provided safety is acceptable. The FAST-TAVI trial evaluated unselected patients discharged early $(n=360$, within $72 \mathrm{~h})$ vs. late $(n=136$, after $72 \mathrm{~h}$ ) after transfemoral TAVI [54]. There were no significant differences in mortality, stroke or re-hospitalisation at 30 days, although the early discharge group had less PPM implantation $(4.1 \%$ vs. $15 \%, p<0.001)$, less major vascular complications $(0.6 \%$ vs. $5.6 \%, p=0.014)$ and numerically less life-threatening bleeding ( $8.9 \%$ vs. $11.1 \%, p=0.465)$. Nevertheless, FAST TAVI suggests that early discharge is feasible and safe in unselected patients undergoing TAVI. 


\section{Other Structural Advances}

While MitraClip (Abbott) is an established (IIaC) intervention for patients with primary $\mathrm{MR}$ and poor LV ejection fraction (LVEF < 30\%) refractory to medical therapy $[33,55]$, its role in secondary mitral regurgitation (MR) was perhaps the most controversial and publicised issue within the structural heart community this year.

MITRA-FR, which randomised 304 patients to MitraClip plus medical therapy $(n=152)$ vs. medical therapy alone $(n=152)$ [56], reported that use of MitraClip was not associated with any reduction in the primary efficacy outcome (54.6\% vs. $51.3 \%, P=0.53)$, in all-cause mortality $(24.3 \%$ vs. $22.4 \%)$ or in unplanned rehospitalisation for $\mathrm{HF}(48.7 \%$ vs. $47.4 \%)$ at 1 year.

In contrast, COAPT, which randomised 614 patients with more than moderate MR and reduced LVEF to MitraClip plus medical therapy $(n=152)$ vs. medical therapy alone $(n=152)$ [57], reported that use of MitraClip was associated with a significant reduction in re-hospitalisations for HF (annualised rate $35.8 \%$ vs. $67.9 \%, P<0.001)$ and reduction in all-cause mortality $(29.1 \%$ vs. $46.1 \%, P<0.001)$ [57].

Of note, patients in COAPT had more severe MR compared with MITRA-FR (effective regurgitant orifice area $41 \mathrm{~mm}^{2}$ vs. $31 \mathrm{~mm}^{2}$ ), smaller mean LV end-diastolic volume $\left(101 \mathrm{~mL} / \mathrm{m}^{2}\right.$ vs. $135 \mathrm{~mL} / \mathrm{m}^{2}$ ) [58] and required patients to be on maximally tolerated medical therapy prior to enrolment which may help explain the outcome differences in COAPT and MITRA-FR. The RESHAPE-HF2 study, estimated to complete recruitment in 2019, may provide further insight into the benefits and role of MitraClip in secondary MR [59].

Percutaneous tricuspid valve repair data were reported from the TRI-REPAIR CE mark study [60] which evaluated the safety and performance of the Edwards Lifesciences Cardioband $^{\mathrm{TM}}$ Tricuspid Valve Reconstruction System (Fig. 3) for annular reduction in 30 patients with moderate to severe functional tricuspid regurgitation. Technical success was $100 \%$. At 30 days, two deaths occurred, one of which was device-related. At discharge there

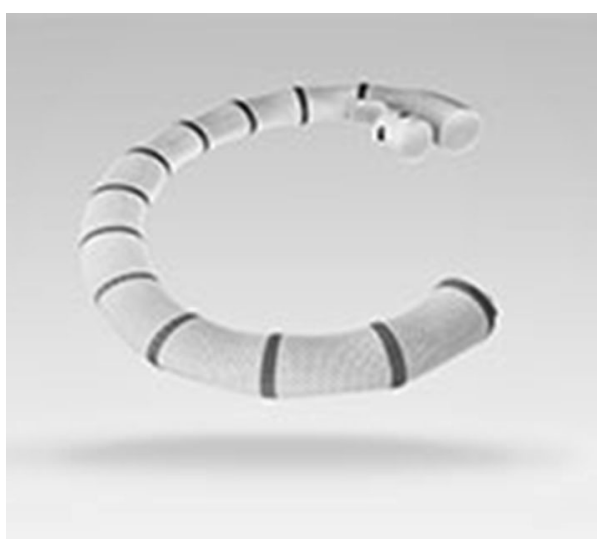

Fig. 3 The semi-rigid incomplete annuloplasty Cardioband. Image permitted and courtesy of Edwards Lifesciences (Irvine, CA, USA)

was a significant reduction in annular diameter (36.9 $\mathrm{mm}$ vs. $43.3 \mathrm{~mm}, P<0.001$ ), effective regurgitant orifice and vena contracta alongside improvement in NYHA class, quality of life scores and 6-min walk test. Longer-term data are awaited.

Several important medication-based studies reported with respect to the management of valvular and structural heart disease. The POET study randomised 400 stabilised patients being treated with intravenous antibiotics for leftsided endocarditis (due to Streptococcus, Enterococcus faecalis, Staphylococcus aureus or coagulase-negative staphylococci) to switch after 10 days to oral antibiotic treatment for up to 6 weeks (201 patients) or to continue intravenous treatment (199 patients) for 6 weeks [61]. Switching to oral antibiotics was non-inferior with respect to the primary composite outcome of all-cause mortality, unplanned cardiac surgery, embolic events or relapse of the primary pathogen at 6 months (9\% vs. $12.1 \%$, $P=0.40$ ) suggesting that such a strategy may be safe, effective and facilitate early hospital discharge.

The management of aortopathy is complex and there are well-published guidelines for when surgical intervention should be considered [62]. The previously described advantageous role of angiotensin II receptor blockers (ARB) in the management of Marfan aortopathy was further supported by outcomes from the 
AIMS study [63] which randomised 192 patients, aged 6-40 years with Marfan syndrome, to irbesartan (150-300 mg) vs. placebo. Of note, $56 \%$ patients also took a beta-blocker during the study. ARB vs. placebo significantly reduced the rate of aortic dilatation at 5 years (0.22 $\mathrm{mm}$ /year relative reduction, $P=0.030)$.

\section{ADVANCES IN CARDIOVASCULAR PREVENTION}

\section{Diabetes and CV Disease}

Previous data from the EMPA-REG OUTCOME study reported that adding empagliflozin to standard care in patients with type 2 diabetes (T2D) was associated with a lower rate of MACE and of all-cause death [64]. The role of the sodium glucose cotransporter-2 (SGLT-2) inhibitor class was examined closely this year with several trials reporting positive results. In the Cardiovascular Events Associated With Sodium Glucose Cotransporter-2 Inhibitors Versus Other Glucose-Lowering Drugs (CVD-REAL 2) trial 470,128 patients with T2D were treated with one of six SGLT-2 inhibitors or another oral glucose-lowering medication [65]. Propensity matched analysis showed that those treated with an SGLT-2 inhibitor had a 49\% lower risk of death (CI 0.37-0.70; $P<0.001$ ) and significantly lower risks of hospitalization for HF, MI or stroke vs. other glucose-lowering medication during follow-up of up to 1 year.

DECLARE TIMI 58 trial randomised 17,160 patients with T2D to dapagliflozin vs. placebo on top of standard care [66]. After an average follow-up of 4.2 years, use of dapagliflozin was associated with a significant reduction in hospital admission for HF (HR 0.73; CI 0.61-0.88), a numerical but non-significant reduction in MACE and no difference in mortality.

The Canagliflozin Cardiovascular Assessment Study (CANVAS) trial previously reported that canagliflozin in T2D was associated with reduction in MACE and reduction in HF events. Further analysis [67] reported that while overall $\mathrm{CV}$ death or hospitalized HF was reduced in those treated with canagliflozin compared with placebo (HR 0.78; 95\% CI 0.67-0.91) across a broad range of patient subgroups, the benefit appeared greater in those with prior history of HF (HR 0.61; 95\% CI 0.46-0.80) vs. those without $\mathrm{HF}$ at baseline (HR 0.87; 95\% CI 0.72-1.06; $P_{\text {interaction }}=0.021$ ).

Although each study showed benefit there were some differences between the results reported (Table 1). All three trials noted a significant reduction in HF outcomes. EMPA-REG and CANVAS reported significant reduction in MACE. Only EMPA-REG reported a significant reduction in CV mortality benefit. Explanations for these results may include differences in selectivity for SGLT-2 vs. SGLT-1 transporters, differences in baseline cardiovascular risk, use of concurrent cardioprotective medications, and methods of CV event adjudication.

The EMPA-Heart Cardiolink-6 study [68] provided some insight into the mechanism of benefit seen with SGLT-2 inhibitors in that by 6 months, those treated with empagliflozin vs. placebo had significantly lower left ventricular mass as measured by MRI and lower systolic blood pressure mean change $(-7.9 \mathrm{mmHg}$ vs. $-0.7 \mathrm{mmHg} ; P=0.03$ ).

Several previous studies have suggested that dipeptidyl peptidase (DPP)-4 inhibitors may be associated with an increased risk of heart failure events [69, 70]. The VIVIDD trial looked at safety of vildagliptin, a DPP-4 inhibitor, in patients with heart failure and ejection fractions of $<40 \%$. In this double-blind study patients were randomised to receive either vildagliptin or placebo and followed up for an average of 52 weeks when they were reassessed with BNP measurement and echocardiogram. There was no significant difference in the adjusted mean change in LVEF of $4.95 \pm 1.25 \%$ in the vildagliptin-treated patients and $4.33 \pm 1.23 \%$ in the placebo-treated patients $(P=0.667)$ [71]. However, left ventricular end-diastolic and end-systolic volumes were found to increase more in the vildagliptin group by $17.1 \mathrm{ml}$ (CI $4.6-29.5 \mathrm{ml} ; P=0.007)$ and $9.4 \mathrm{ml}(95 \% \mathrm{CI}$ -0.49 to $19.4 \mathrm{ml} ; P=0.062$ ), respectively. These results suggest that DPP-4 inhibitors are unlikely to be the optimal anti-hyperglycaemic therapy for patients with established heart failure; however, additional clinical trials are needed to firmly establish the benefits and risks. 
Table 1 Comparison of the major trials examining CV outcomes with SGLT-2 inhibitors

\begin{tabular}{llll}
\hline & $\begin{array}{l}\text { Empagliflozin } \\
\text { EMPA-REG }\end{array}$ & $\begin{array}{l}\text { Canagliflozin } \\
\text { CANVAS }\end{array}$ & $\begin{array}{l}\text { Dapagliflozin } \\
\text { DECLARE }\end{array}$ \\
\hline CV death, MI, stroke & $\downarrow$ & $\downarrow$ & $\rightarrow$ \\
CV death & $\downarrow$ & $\rightarrow$ & $\rightarrow$ \\
Heart failure admissions & $\downarrow$ & $\downarrow$ & $\downarrow$ \\
Subjects with established CV disease & $99 \%$ & $66 \%$ & $41 \%$ \\
Average age & 63 & 63 & 64 \\
\hline
\end{tabular}

\section{Hypertension}

Health promotion remains a constant challenge, particularly getting meaningful promotion messages to groups most at risk. In a novel approach for blood pressure (BP) assessment, the Barbershop trial randomised 319 men in a non-traditional healthcare setting (52 blackowned barbershops) to in-house pharmacist-led intervention (to measure and manage BP) vs. active control (in which barbers encouraged lifestyle modification and doctor's appointments) [72]. By 6 months the intervention group had a greater reduction in mean BP $(-27.0 \mathrm{mmHg}$ vs. $-9.3 \mathrm{mmHg}$, CI $14.7-28.4$; $P<0.001)$ and more patients achieved a BP target of $130 / 80 \mathrm{mmHg} \quad(63.6 \%$ vs. $11.7 \%$; $P<0.001)$.

Conventional hypertension treatment algorithms typically use a stepwise approach that can lead to delay in achieving BP goals. The TRIUMPH study randomised 700 patients with hypertension (baseline mean BP 154/90 mmHg, $59 \%$ not on treatment) to a low-dose triple combination polypill (telmisartan $20 \mathrm{mg}$, amlodipine $2.5 \mathrm{mg}$ and chlorthalidone $12.5 \mathrm{mg}$ ) vs. standard care [73]. The BP treatment target was $<140 / 90 \mathrm{mmHg}$ or $<130 / 80 \mathrm{mmHg}$ in diabetes and chronic kidney disease. By 6 months, those assigned to a polypill approach had a lower mean BP $(125 / 76 \mathrm{mmHg}$ vs. $134 / 81 \mathrm{mmHg}$ ) and significantly more achieved their BP target (70\% vs. 55\%; $P<0.001$ ).

The role of catheter-based renal denervation (RDN) in the management of hypertension has been uncertain. Although several early unblinded studies reported significant BP reductions following $\mathrm{RDN}$, the large SYMPLICITY HTN3, which randomised patients to RDN with a single-electrode catheter vs. a sham procedure, did not show significant benefit. However, SYMPLICITY HTN3 was criticised for several reasons such as limited extent of denervation, inexperienced operators, nonadherence to medication and patient selection may have influenced results [8] and the SPYRAL HTN OFF MED trial in patients with mild-moderate BP not on antihypertensives showed that RDN using the multi-electrode spyral catheter $(n=38$; mean 44 ablations per patient; 18 main artery, 26 branch artery; Fig. 4) vs. sham control $(n=42)$ had subsequently confirmed modest reductions in office and ambulatory BP (providing proof of concept for renal denervation) [8]. Most recently, the SPYRAL HTN-ON MED trial randomised 80 patients with poorly controlled hypertension on up to three drugs to contemporary RDN (mean ablations $45.9 \pm 13.7$, main arteries treated $2.3 \pm 0.5$, branches treated $5.8 \pm 2.2$ ) vs. sham [74]. By 6 months, patients in the RDN group had significantly greater reduction in mean 24 -h systolic BP $(9.0 \mathrm{mmHg}$ vs. $1.6 \mathrm{mmHg} ; P=0.005)$ confirming that RDN may also be of benefit for patients with resistant hypertension already on treatment.

The Anglo-Scandinavian Cardiac Outcomes Trial (ASCOT) Legacy Study gave an insight into the long-term CV and mortality outcomes of blood pressure and lipid-lowering treatments [75]. ASCOT randomised 8580 patients with hypertension to amlodipine-based vs. atenolol- 


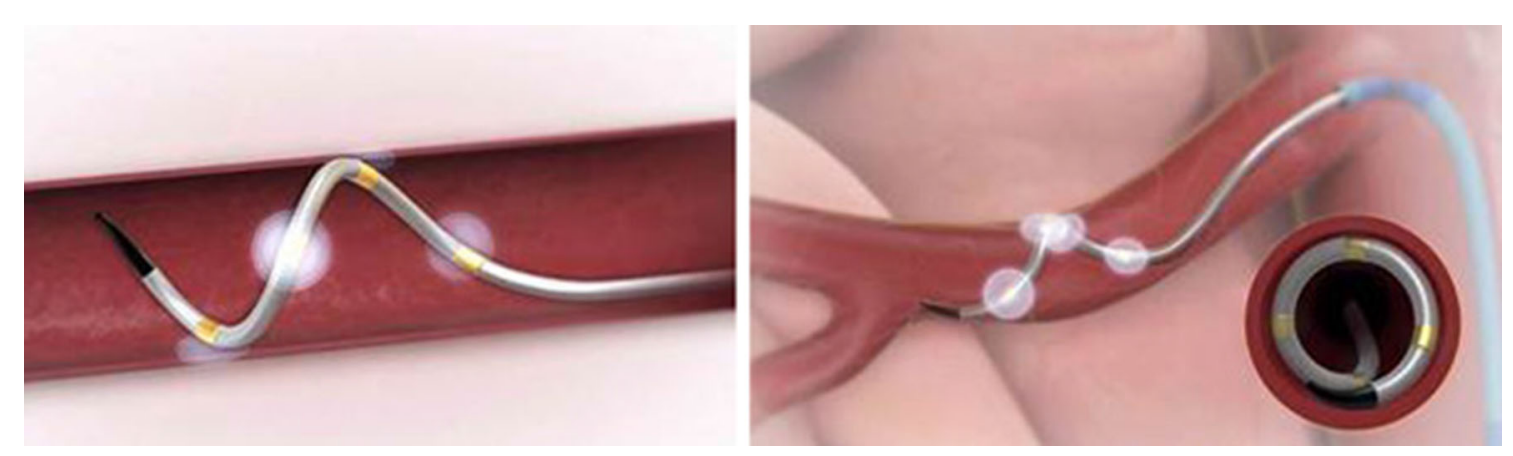

Fig. 4 Symplicity Spyral multi-electrode catheter. Image used with the permission of Medtronic. (c) 2013 Medtronic

based BP-lowering treatment and, in a $2 \times 2$ design, those with total cholesterol $\leq 6.5 \mathrm{mmol} / \mathrm{L}(n=4605)$ were randomised to atorvastatin vs. placebo. At a median followup of 15.7 years, those receiving amlodipine vs. atenolol had significantly fewer strokes (adjusted HR 0.71; CI 0.53-0.97; $P=0.0305$ ), and in the lipid-lowering group, those receiving atorvastatin vs. placebo had significantly lower CV mortality (HR 0.85; CI 0.72-0.99, $P=0.0395)$ confirming the long-term advantages of contemporary BP-lowering strategies and preventative lipid-lowering therapy in a hypertensive population.

\section{Aspirin in Primary Prevention}

The role of aspirin in primary prevention has been unclear. Early trials showed small-tomodest $\mathrm{CV}$ benefits in high-risk patients, but they were conducted at a time when risk factor control was suboptimal. Two large studies evaluated aspirin further in this setting. In the Aspirin to Reduce Risk of Initial Vascular Events (ARRIVE) trial, 12,546 patients deemed to be at moderate $\mathrm{CV}$ risk were randomised to $100 \mathrm{mg}$ aspirin vs. placebo [76]. Those at high risk of gastrointestinal bleeding were excluded. During a mean follow-up of 7.4 years, aspirin was not associated with any significant reduction in $\mathrm{CV}$ events (4.29\% vs. $4.48 \%$, HR 0.96; CI 0.81-1.13; $P=0.6083)$ but was associated with significantly more gastrointestinal bleeding $(0.97 \%$ vs. $0.46 \%$; HR 2.11; CI 1.36-3.28; $P=0.0007$ ). Allcause mortality was neutral between the two groups (HR 0.99; 95\% CI 0.80-1.24; $P=0.95$ ).
The ASCEND trial randomised 15,480 patients with diabetes but no known CV disease to aspirin $100 \mathrm{mg}$ vs. placebo [77]. Aspirin was associated a $12 \%$ reduction in the composite of vascular death, MI or stroke/transient ischemic attack $(8.5 \%$ vs. $9.6 \%$, rate ratio 0.88 ; CI $0.79-0.97 ; P=0.01$ ) but this was offset by a $29 \%$ increase in rate of major bleeding events $(4.1 \%$ vs. $3.2 \%$, rate ratio 1.29 ; CI $1.09-1.52$; $P=0.003$ ) with most of the excess being gastrointestinal bleeding and other extracranial bleeding. All-cause mortality was neutral between the trial groups (rate ratio, 0.94; 95\% CI 0.85-1.04) and there was no significant difference in the incidence of gastrointestinal tract cancer. Thus while aspirin has an established benefit in secondary prevention, these large trials do not support a routine role for aspirin in primary prevention.

\section{Lipids}

Results from the Fourier trial published in 2017 were promising, with reduced LDL-C levels and reduced $\mathrm{CV}$ events in a group of stable patients with established CVD [78]. The ODYSSEY outcomes trial built on this by examining the use of alirocumab, a human monoclonal antibody to proprotein convertase subtilisin-kexin type 9 (PCSK9) following ACS [79]. ODYSSEY randomised 18,924 patients with ACS in the past year, already taking maximal tolerated statin therapy, and suboptimal lipid control (LDL$\mathrm{C} \geq 1.8 \mathrm{mmol} / \mathrm{l}$, non $-\mathrm{HDL}-\mathrm{C} \geq 2.6 \mathrm{mmol} / \mathrm{l}$ or apolipoprotein $\mathrm{B} \geq 80 \mathrm{mg} / \mathrm{dl}$ ) to alirocumab (achieving mean LDL-C $1.0 \mathrm{mmol} / \mathrm{l}$ ) vs. placebo 
(mean LDL-C $2.4 \mathrm{mmol} / \mathrm{l}$ ). After a median follow-up of 2.8 years, alirocumab was associated with a significant reduction in the composite primary endpoint of death from coronary heart disease, MI, ischaemic stroke or unstable angina requiring hospitalisation (HR 0.85; CI $0.78-0.93 ; P<0.001$ ) driven mainly by non-fatal events. There was a numerical reduction in all-cause death (3.5\% vs. $4.1 \%)$, although it was ineligible for significance testing on hierarchical analysis. Benefits were greatest in those with baseline LDL-C $\geq 2.6 \mathrm{mmol} / \mathrm{l}$.

The role of omega-3 fatty acid (FA) supplementation has been controversial. Two important trials in the past year have added to the debate.

The ASCEND trial (in addition to its aspirin evaluation above) randomised the 15,480 diabetic patients with no known CVD to $1 \mathrm{~g}$ omega-3 FA eicosapentaenoic acid (EPA) and docosahexaenoic acid (DHA) vs. placebo (olive oil) [80]. At a mean of 7.4 years treatment, omega-3 FA did not reduce the composite of vascular death/MI/stroke/TIA ( $8.9 \%$ vs. $9.2 \%$, rate ratio $0.97 \%$; CI $0.87-1.08 ; P=0.55$ ). These findings were in keeping with other recent data testing low doses of combined EPA/DHA omega-3 FAs in unselected patients.

Given that higher-dose, EPA-only supplementation was previously reported to be of benefit on top of statin therapy in a Japanese population [81], the Reduction of Cardiovascular Events with Icosapent Ethyl-Intervention Trial (REDUCE-IT) trial randomised 8179 patients with CVD or diabetes and other risk factors, who despite statin therapy (and LDL$\mathrm{C}<2.6 \mathrm{mmol} / \mathrm{l}$ ) had residual elevation in triglycerides (baseline median $2.4 \mathrm{mmol} / \mathrm{l}$ ) to a total of $4 \mathrm{~g}$ ( $2 \mathrm{~g}$ bd) of icosapent ethyl, a highly purified EPA ethyl ester vs. placebo [82]. EPA reduced triglycerides by $18 \%$ from baseline. At a mean follow-up of 4.9 years, EPA was associated with a $25 \%$ reduction in the composite endpoint of CV death, MI, stroke, coronary revascularisation or unstable angina $(17.2 \%$ vs. $22 \%$, HR 0.75; CI 0.68-0.83; $P<0.001$ ) and a $20 \%$ reduction in the secondary endpoint of $\mathrm{CV}$ death $(4.3 \%$ vs. 5.2\%; HR $0.80 ; \quad 95 \% \quad \mathrm{CI}$ $0.66-0.98 ; \quad P=0.03)$ but increased serious bleeding events $(2.7 \%$ vs. $2.1 \% ; P=0.06)$.
Potential mechanisms of $\mathrm{CV}$ benefit include triglyceride reduction (although the CV benefit was more than would be anticipated from the degree of triglyceride reduction alone), membrane stabilisation, coronary plaque stabilisation/regression and possibly an antiinflammatory effect reflected by reduction in CRP. Thus after several disappointing omega-3 FA trials, REDUCE-IT suggests that icosapent ethyl may be beneficial at least in those with residual elevation in triglycerides despite statincontrolled LDL-C levels.

\section{Weight Management}

Lorcaserin is a selective serotonin $2 \mathrm{C}$ receptor agonist that has been shown to be useful for weight management in overweight and obese patients, but its CV safety had not been fully evaluated. The CAMELLIA-TIMI 61 study [83] randomised 12,000 overweight and obese patients to lorcaserin vs. placebo on top of standard care. At 1 year, more patients receiving lorcaserin achieved weight loss of at least 5\% $(38.7 \%$ vs. $17.4 \%$, OR 3.01 ; CI $2.74-3.30$; $P<0.001)$. By a median follow-up of 3.3 years there was no significant increase in major $\mathrm{CV}$ events $(4.1 \%$ vs. $4.2 \%)$ suggesting that lorcaserin appears safe to use in patients with $\mathrm{CV}$ risk factors.

\section{Coronary Imaging}

In the Scottish computed tomography of the heart (SCOT-HEART) trial 4146 patients with stable chest pain were randomised to either CT coronary angiography (CTCA) and standard care or standard care alone [84]. In a follow-up of 4.8 years, those randomised to CTCA had a lower rate of CHD death or MI $(2.3 \%$ vs. $3.9 \%$, HR $0.59 ; \quad$ CI $0.41-0.84 ; \quad P=0.004)$. This improved outcome is likely related to CTCA leading to a reclassification of diagnosis in around 1 in 4 patients and change in management in around 1 in 6 including greater prescription of preventive medications $(19.4 \%$ vs. 14.7\%; OR 1.40; CI 1.19-1.65) Of interest, and although performed earlier, the final overall rate of invasive coronary angiography with or 
without revascularisation was not significantly different between groups.

\section{Vascular Inflammation}

Given the previous finding that reducing vascular inflammation using IL-1 $\beta$ inhibitor canakinumab in the CANTOS trial reduced $\mathrm{CV}$ events [85] independent of LDL-C, there was significant interest in results of the Cardiovascular Inflammation Reduction Trial (CIRT) which randomised 4768 patients with established coronary disease and either diabetes or metabolic syndrome to low-dose methotrexate vs. placebo [86]. Disappointingly, the trial was stopped early for futility after a median of 2.3 years when low-dose methotrexate was not found to reduce levels of IL-1 $\beta$, IL-6, CRP or CV events. Differences between CIRT and CANTOS data are instructive. Firstly, patients in CIRT did not have evidence of inflammation or elevated CRP (baseline $1.6 \mathrm{mg} / \mathrm{l}$ ) whereas elevated CRP was an inclusion criteria in CANTOS (baseline $4.2 \mathrm{mg} / \mathrm{l})$. Secondly, trials of several other antiinflammatory agents (e.g. the phospholipase inhibitor darapladib or the p38 MAP kinase blocker losmapimod) have been disappointing; it is thus possible that IL- $1 \beta-$ IL- 6 signalling, a process initiated at the NLRP3 inflammasome, is central to atherosclerosis development and specific targeting is required. Agents such as colchicine and oral NLRP3 inhibitors that may also affect this pathway are currently under investigation.

\section{ADVANCES IN ACS AND ANTITHROMBOTIC THERAPY}

The optimal duration of dual antiplatelet therapy (DAPT) in patients with ACS is often debated, but current guidelines support at least 12 months [87]. The SMART-DATE trial [88] randomised 2712 patients following ACS to short 6-month DAPT duration vs. 12 months or longer. Clopidogrel was the $\mathrm{P}_{2} \mathrm{Y}_{12}$ inhibitor in approximately $80 \%$ of patients and all were treated with a second- or third-generation DES (Resolute Integrity, Medtronic Vascular; Xience
Prime, Abbott Vascular; or BioMatrix Flex, Biosensors Inc). Shorter-duration DAPT was associated with a similar incidence of the primary composite endpoint of all-cause mortality, MI and stroke $(4.7 \%$ vs. $4.2 \%, P=0.03)$, but increased incidence of MI $(1.8 \%$ vs. $0.8 \%$, $P=0.02)$ potentially related to the numerically higher stent thrombosis rates $(1.1 \%$ vs. $0.7 \%$, $P=0.32$ ). SMART-DATE thus supports guidelines retaining a 12-month recommendation for DAPT post ACS.

Aspirin is established as the baseline antiplatelet therapy in guidelines; however, given the excellent inhibition of platelet aggregation seen with ticagrelor, it has been queried whether ticagrelor monotherapy might be an adequate substitute for DAPT. The GLOBAL LEADERS trial randomised 15,968 patients with ACS or stable CAD following PCI with a Biolimus A9-eluting stent to a novel treatment arm of 1 month only DAPT (aspirin and ticagrelor) followed by 23 months ticagrelor monotherapy vs. 12 months DAPT (aspirin and ticagrelor or clopidogrel) followed by 12 months aspirin monotherapy [89]. At 2 years there was a trend towards fewer patients reaching the primary composite endpoint of death or new Q wave MI in the ticagrelor monotherapy group but this did not reach clinical significance $(3.81 \%$ vs. $4.37 \%$, rate ratio $0.87,95 \%$ CI $0.75-1.01)$. The bleeding risk between the two groups was similar $(2.04 \%$ vs. $2.02 \%, P=0.77)$. Although the 2-year results were disappointing, in exploratory analysis, at 12 months death or QMI was significantly reduced in the ticagrelor monotherapy arm $(1.95 \%$ vs. $2.47 \% ; P=0.028)$ and drug adherence was lower with ticagrelor vs. aspirin between 12 and 24 months. We thus await studies such as TWILIGHT investigating ticagrelor monotherapy further.

Prior studies including ARMYDA and Naples II have suggested benefit with statin loading prior to elective PCI $[90,91]$. The SECURE-PCI trial [92] randomised 4191 patients with ACS and planned invasive management to atorvastatin $80 \mathrm{mg}$ vs. placebo before and $24 \mathrm{~h}$ after invasive assessment. Both groups later received atorvastatin $40 \mathrm{mg}$ for 30 days. At 30 days, there was only a non-significant trend toward a reduction in the risk of MACE (all-cause 
mortality, acute MI, stroke and unplanned coronary revascularization) in the atorvastatin preloading arm vs. placebo (HR 0.88; 95\% CI $0.69-1.11)$. However, only $64.7 \%$ of the cohort went on to have PCI after invasive assessment and in this subgroup and a significant reduction in MACE with atorvastatin preloading was seen (6.0\% vs. $8.2 \%$, HR 0.72 , CI $0.54-0.96)$ suggesting that further prospective studies in such patients may be useful.

A lower threshold for diagnosing $\mathrm{MI}$ has been permitted by using high sensitivity troponin assays, but whether this translates into improved use of secondary prevention and/or improved clinical outcomes is unknown. In the High STEACS trial, use of a high sensitivity troponin assay reclassified $17 \%$ patients as MI or myocardial injury. However, only one-third of these patients had a subsequent diagnosis of type $1 \mathrm{MI}$ and identifying this did not translate into a lower incidence of recurrent $\mathrm{MI}$ or $\mathrm{CV}$ death at 1 year [93]. These results may due to the relatively small number reclassified as type 1 MI and lack of clear guidelines on the ideal management of the patients with type $2 \mathrm{MI}$.

Despite guideline recommendations, there has been no randomised controlled trial evaluating oral anticoagulation (OAC) alone vs. OAC plus single antiplatelet (SAPT) in patients with AF beyond 1-year post PCI. It is an important question, as there are known to be increased bleeding risks with OAC plus SAPT [94]. The OAC ALONE trial randomised patients to OAC alone vs. OAC plus SAPT (stopping earlier than planned after 696 patients because of slow enrolment). The primary endpoint (composite of all-cause death, MI, stroke or systemic embolism) appeared similar in the two groups $(15.7 \%$ vs. $13.6 \%)$ but unfortunately the trial could not confirm non-inferiority because of a lack of power from the small sample size [95].

\section{ADVANCES IN ATRIAL FIBRILLATION AND ELECTROPHYSIOLOGY}

The year 2018 saw both positive and negative trial outcomes with respect to catheter ablation for atrial fibrillation (AF), brady-pacing and ventricular arrhythmia therapies.
The CABANA (Catheter ABlation vs. ANtiarrhythmic drug therapy in Atrial fibrillation) study compared the primary outcome of death, bleeding, serious stroke, or cardiac arrest at 5 years for patients undergoing AF ablation $(n=1108) \quad$ vs. antiarrhythmic therapy ( $n=1096)$ alone [96]. There was no significant difference in the primary endpoint ( $8 \%$ vs. $9.2 \%, P=0.3)$ in an "intention-to-treat" analysis. However, over $25 \%$ of the medication arm crossed over and underwent ablation and there was a significant difference in the "as-treated" analysis with significant reduction in the primary endpoint (7\% vs. $10.9 \%, P=0.006)$ and death $(4.4 \%$ vs. $7.5 \%, P=0.005)$. Given the high crossover, the as-treated analysis is likely to be more representative of treatment effect, although the factors leading to the high crossover were not fully explained. Of note, very few severe complications were reported suggesting good case selection and, overall, CABANA suggests that AF ablation in the right patient may be superior to medical therapy alone. However, further research, ideally compared with a sham control, is still needed [97].

Idarucizumab is well established for reversal of the direct thrombin inhibitor dabigatran. Interim analysis from ANNEXA-4, investigating the effects of andexanet, a factor Xa decoy protein, on bleeding and anti-factor Xa activity in patients presenting with an acute major bleed, showed that good or excellent haemostasis was achieved in $83 \%$ of the study population ( $n=137$ efficacy population) [98]. In the safety population $(n=227)$, ischaemic stroke, MI and venous thromboembolism (VTE) occurred in $2.6 \%$ and $11 \%$ of patients at 3 days and 30 days, respectively. All patients had OAC stopped on presentation and only $57 \%$ had OAC restarted by 30 days.

If $\mathrm{OAC}$ is inadequate, the risk of periprocedural thromboembolism with DC cardioversion (DCC) may be greater than 5\%, whereas use of OAC e.g. by vitamin $\mathrm{K}$ antagonist (VKA) for at least 3 weeks prior to, during and at least 4 weeks post DCC reduces the risk to below 1\% and is thus the guideline recommended timing except with early presentation $(<48 \mathrm{~h}$ post $\mathrm{AF}$ onset), or if imaging is undertaken to exclude to left atrial thrombus. The EMANATE trial, which 
provided the first prospective data for apixaban in DCC, included evaluation of a novel loading dose strategy and randomised 1500 patients with recent onset AF (2/3 new onset, $78 \%$ within 3 months) and at most $48 \mathrm{~h}$ prior OAC (2/3 with no prior OAC) to short duration apixaban (usual dosing schedule, \pm loading dose at clinician discretion to achieve rapid steady state and allow earlier DCC) (5-10 mg) vs. short duration heparin/VKA [99]. Pre-procedural imaging was not mandated but was obtained in 855 patients (mainly by TOE); of these, 61 had evidence of left atrial thrombus and thus DCC was deferred and imaging was repeated approximately 1 month later by which time thrombi had resolved in $52 \%$ of apixaban and $56 \%$ of heparin/VKA groups, respectively. In total, 1038 had active cardioversion (DCC and/or pharmacological), 300 had spontaneous cardioversion and 162 patients were not cardioverted.

Stroke and bleeding outcomes appeared similar in the active cardioversion patients (Table 2) and in the full intention to treat population, although the trial was underpowered (approx. 48,000 patients would be required to power non-inferiority). Nevertheless, results are consistent with other factor Xa inhibitor studies and suggest that apixaban is a viable alternative to heparin/VKA in patients undergoing early or elective DCC. A short duration apixaban loaded strategy appears reasonable in selected patients but does not obviate the need for transoesophagael echocardiography, since imaging identified $7 \%$ of patients with thrombi, of which only about half had resolved by 1 month.

Implantable cardiac defibrillator implantation has a strong guideline recommendation in patients with persistently low EF $(<35 \%)$ following an interim period of up to 3 months after MI [100, 101]. The VEST study evaluated whether a wearable cardiac defibrillator (WCD) (Fig. 5) would reduce the incidence of sudden death during the initial high-risk period after MI [102]. A total of 1524 patients were randomly assigned (2:1) to WCD and 778 to a control group. At 90 days, use of a WCD did not lead to a significant reduction in the primary endpoint outcome of arrhythmic death $(1.6 \%$ vs. $2.4 \%, P=0.18$ ). Despite this, WCD was surprisingly associated with a $36 \%$ reduction in a secondary endpoint of all-cause death $[3.1 \%$ vs. $4.9 \%$ (nominal $P=0.04$ )]. Given that there is no clear mechanism for the WCD to influence nonarrhythmic death, it is possible that some arrhythmic deaths were misclassified as nonarrhythmic deaths; alternatively, it could merely be a chance finding. From a practical perspective, WCD compliance was less than optimal at a median of $18 \mathrm{~h}$ /day and only 12 of 48 patients in the WCD group were wearing the device at time of death. Thus, the role of WCD post $\mathrm{MI}$ remains unclear and further research is required.

Ventricular tachycardia (VT) refractory to drug therapy or catheter ablation is typically an end-stage process with limited palliative options. The ENCORE-VT single-arm trial

Table 2 Time delay to DC cardioversion and outcomes in patients undergoing active cardioversion

\begin{tabular}{|c|c|c|c|c|c|c|}
\hline \multirow[t]{2}{*}{$\begin{array}{l}\text { Active cardioversion } \\
(n=1038)\end{array}$} & \multicolumn{2}{|c|}{$\begin{array}{l}\text { Apixaban } \\
\text { Loading dose }(n=272)\end{array}$} & \multicolumn{2}{|c|}{$\begin{array}{l}\text { Apixaban } \\
\text { No loading dose }(n=247)\end{array}$} & \multicolumn{2}{|c|}{ Heparin/VKA $(n=519)$} \\
\hline & $\begin{array}{l}\text { With } \\
\text { imaging }\end{array}$ & $\begin{array}{l}\text { Without } \\
\text { imaging }\end{array}$ & $\begin{array}{l}\text { With } \\
\text { imaging }\end{array}$ & $\begin{array}{l}\text { Without } \\
\text { imaging }\end{array}$ & $\begin{array}{l}\text { With } \\
\text { imaging }\end{array}$ & $\begin{array}{l}\text { Without } \\
\text { imaging }\end{array}$ \\
\hline Number & 238 & 34 & 155 & 92 & 407 & 111 \\
\hline Mean time (days) & 3.3 & 4.1 & 21.7 & 32.5 & 11.5 & 40.7 \\
\hline Median time (days) & 1 & 1 & 15 & 30 & 2 & 43 \\
\hline Strokes & 0 & 0 & 0 & 0 & 3 & 0 \\
\hline Major bleeding & 0 & 1 & 1 & 1 & 2 & 0 \\
\hline
\end{tabular}




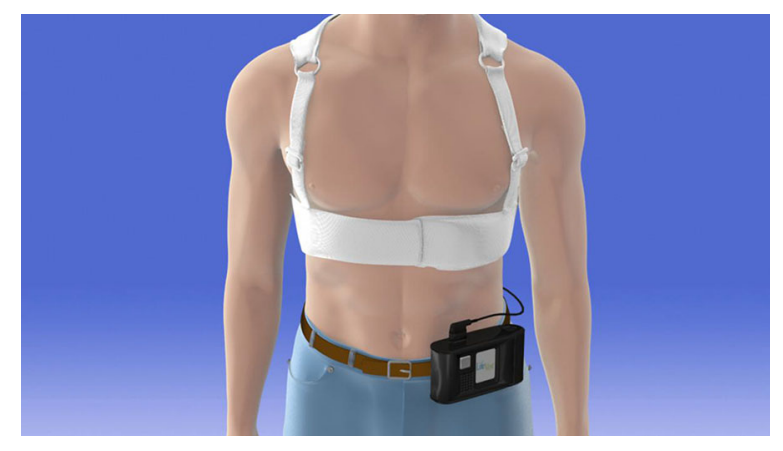

Fig. 5 The LifeVest device, Zoll Medical

assessed the effectiveness and safety of a novel electrophysiology (EP)-guided non-invasive stereotactic body radioablation (SBRT) in 19 patients with drug or catheter-ablation refractory VT or premature ventricular contractions [103]. SBRT ablation was associated with a reduction in the median number of VT episodes 6 months before vs. 6 months after the procedure (119 vs. 3$)$ and decrease in median ICD shocks (4 vs. 0). Complications included clinical pericarditis $(n=1)$, radiation pneumonitis $(n=2)$ and asymptomatic pericardial effusion $(n=5)$. The mechanism of benefit is not entirely clear-the VT disappeared much faster than expected from the radiation effects alone (since radiation typically takes weeks to months to cause fibrosis). Nevertheless, SBRT thus appears a potential effective therapy for resistant VT but further research, in a larger population, is needed to formally assess the efficacy and safety.

Biventricular pacing (BiVP) has revolutionised the treatment of HF in patients with sinus rhythm and left bundle branch block but left ventricular lead placement is not always technically possible. Furthermore, BiVP does not fully normalise ventricular activation and, therefore, the ventricular resynchronisation is imperfect. Right ventricular pacing (RVP) for bradycardia may cause or worsen HF in some patients by causing dyssynchronous ventricular activation. His bundle pacing (HBP) activates the ventricles via the native His-Purkinje system, resulting in true physiological pacing, and thus may be promising for bradycardia pacing, possibly traditional CRT indications, and potentially new indications therapy in $\mathrm{HF}$ pacing such as patients with PR prolongation but narrow QRS. In a non-randomised study, HBP ( $n=304 / 332$ attempted) was compared with RVP $(n=433)$ for patients undergoing bradycardia pacing [104]. HBP was associated a lower incidence in the composite primary endpoint of death, HF hospitalization or upgrade to $\operatorname{BiVP}(25 \%$ vs. $32 \%, P=0.02)$, with the greatest differences seen in patients with ventricular pacing greater than $20 \%(25 \%$ vs. $36 \%$, $P=0.02$ ) suggesting that $\mathrm{HBP}$ is feasible and may be superior to RVP. Larger randomised trials are being planned.

\section{ADVANCES IN HEART FAILURE}

Sacubitril-valsartan has been shown to be superior to enalapril in patients with chronic HF [105]. A new study (Comparison of Sacubitril-Valsartan Versus Enalapril on Effect on NTproBNP in Patients Stabilized From an Acute HF Episode, PIONEER-HF) randomised 887 patients presenting with acute decompensated HF (plus LVEF $\leq 40 \%$ within the prior 6 months and raised BNP levels) to sacubitril-valsartan vs. enalapril [106]. By 8 weeks, sacubitril-valsartan was associated with an $84 \%$ greater reduction in NT-proBNP vs. enalapril ( $-46.7 \%$ vs. $-25.3 \%$; $P<0.001)$ with a difference emerging after 1 week (Fig. 6), a $44 \%$ reduction in HF rehospitalisation (ratio 0.56; CI 0.37-0.84) and nonsignificant reduction in death (ratio 0.66; CI $0.30-1.48$ ). PIONEER-HF thus suggests that it is safe to commence sacubitril-valsartan during hospitalisation with acutely decompensated HF once stabilised.

Use of the CardioMEMS device, an implantable pulmonary artery pressure monitor which gives continuous real-time monitoring of the pulmonary artery pressure to help guide diuretic and vasodilator titration, has previously been shown to be associated with reduced hospitalisation in NYHA class 3 HF patients [107] (Fig. 7).

New data from a retrospective, observational 1:1 matched case-control study reported use of the device to be associated with a $30 \%$ reduction in mortality vs. control (0.22 vs. 0.30 ; HR $0.70, \quad$ CI $0.59-0.83, \quad P<0.0001) \quad$ [108]. A 
Weeks Since Randomization

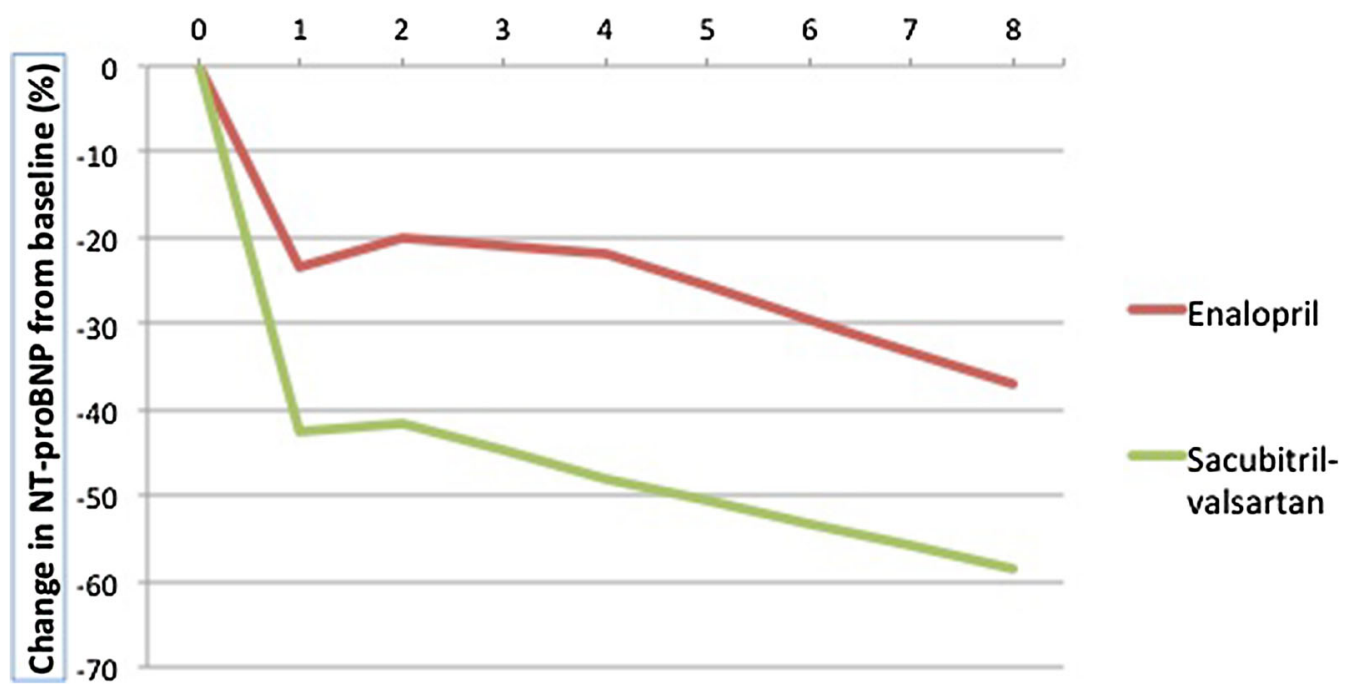

Fig. 6 Reduction in NT pro-BNP seen with sacubitril-valsartan compared with enalapril in PIONEER-HF

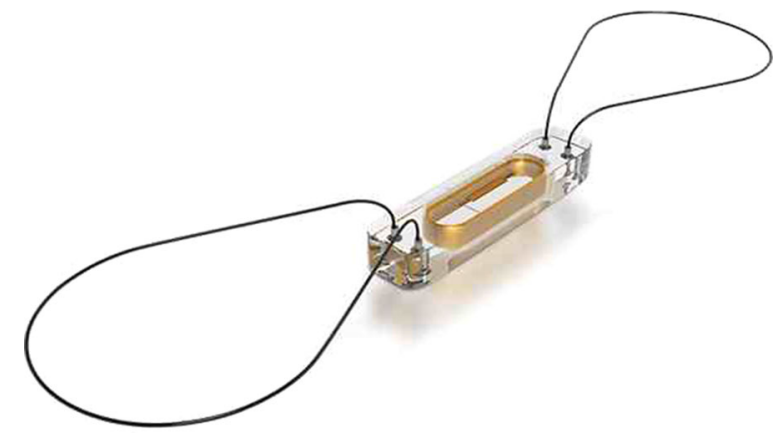

Fig. 7 CardioMEMS device. CardioMEMS is a trademark of Abbott or its related companies. Reproduced with permission of Abbott, (C) 2018. All rights reserved

randomised control trial, GUIDE-HF, is now planned to examine this association more closely [109].

Non-invasive data provided by remote monitoring may also help guide heart failure treatment titration. The Telemedical Interventional Management in Heart Failure II (TIMHF2) [110] randomised 1571 patients with NYHA II or III HF, hospital admission for HF within 12 months and LVEF $\leq 45 \%$ (or higher if oral diuretics being used) to remote patient management (including blood pressure, electrocardiography, weight and oxygen saturation) plus standard care vs. standard care alone. At 1-year follow-up, remote management was associated with a $20 \%$ reduction in days lost due to unplanned CV hospitalisation or all-cause mortality $(4.88 \%$ vs. $6.64 \%$; ratio 0.80 ; CI $0.65-1.00 ; P=0.046)$. Of note, TIM-HF2 excluded depressed patients, which is important, as patient motivation is a key factor in obtaining ongoing quality remote data.

The HeartMate II LV assist device has been associated with improved outcomes in refractory HF but its axial-flow pump design has been associated with device-related complications. The MOMENTUM3 trial [111] randomised 366 patients eligible for $\mathrm{LV}$ assist device to the redesigned HeartMate 3 (using a magnetically levitated centrifugal continuous-flow circulatory pump) $(n=190)$ vs. HeartMate II $(n=176)$. HeartMate 3 was associated with a reduced need for reoperation for malfunction [3 (1.6\%) vs. 30 $(17 \%) ; P<0.001]$ and a lower rate of overall stroke (10.1\% vs. $19.2 \%$, HR 0.47; CI 0.27-0.84, $P=0.02)$ although rates of death and disabling stroke were similar.

Previous studies have reported benefit for early detection and prompt treatment of chemotherapy-related cardiotoxicity with ACE inhibitors and beta-blockers [112, 113]. The Carvedilol for Prevention of Chemotherapy-Induced Cardiotoxicity (CECCY) trial randomised 
192 patients with normal LVEF and HER2-negative breast cancer undergoing anthracyclinebased chemotherapy to carvedilol vs. placebo [114]. At 6 months, use of carvedilol was not associated with benefit in LV systolic function ( $\geq 10 \%$ drop in LVEF; $14.5 \%$ vs. $13.5 \% ; P=1.0$ ) but was associated with a lower incidence of troponin I elevation $(26.0 \%$ vs. $41.6 \%$; $P=0.003)$ and lower incidence of diastolic dysfunction $(28.5 \%$ vs. $37.2 \% ; P=0.039)$. Carvedilol may thus have a modest myocardial protective effect but further studies are needed.

Chronic HF is associated with activation of thrombin-related pathways and thus it was hypothesized that low-dose anticoagulation (shown of benefit in ACS) to reduce thrombin production may improve outcomes. COMMANDER-HF randomised 5022 patients with chronic HF (LVEF $\leq 45 \%$ ), sinus rhythm and stable coronary disease to rivaroxaban $2.5 \mathrm{mg}$ twice daily vs. placebo [115]. At median followup of 21.1 months, rivaroxaban was not associated with any difference in the primary composite endpoint of all cause death, MI or stroke (25.0\% vs. $26.2 \%$; HR 0.94; CI 0.84-1.05; $P=0.27)$ or all-cause death $(21.8 \%$ vs. $22.1 \%$; HR 0.98; CI 0.87-1.10).

Transthyretin amyloid cardiomyopathy is an uncommon but catastrophic cardiomyopathy (mean survival of 2.5 years) due to hereditary or acquired mutations in the TTR gene leading to abnormal myocardial deposition of transthyretin, a protein that normally transports thyroxine and retinol, and a build-up of amyloid bodies. At present management is limited to supportive care. The Efficacy and Safety of Tafamidis in Transthyretin Amyloid Cardiomyopathy (ATTR-ACT) study randomised 441 patients $(2: 1: 2)$ to tafamidis $80 \mathrm{mg}$, tafamidis $20 \mathrm{mg}$ vs. placebo [116]. At 30 months, the pooled tafamidis group was associated with a $30 \%$ reduction in all-cause mortality $(29.5 \%$ vs. 42.9\%; HR 0.70; CI 0.51-0.96), fewer CV-related hospitalisations (0.48 per year vs. 0.70 per year; CI 0.56-0.81), a lower rate of decline in 6-min walk test $(P<0.001)$ and lower rate of decline in KCCQ-OS score $(P<0.001)$. There were no significant differences in numbers and types of adverse events in either group. This trial offers an exciting new possibility of a treatment for patients with a disease that until now has been incurable.

\section{CONCLUSIONS}

This article has highlighted and summarised the key trials that were published and presented in the field of cardiology during 2018. Many of these studies will help guide clinical practice guideline updates and others have shown encouraging early data to guide further drug or device development.

\section{ACKNOWLEDGEMENTS}

Funding. No funding or sponsorship was received for this study or publication of this article.

Authorship. All named authors meet the International Committee of Medical Journal Editors (ICMJE) criteria for authorship for this manuscript, take responsibility for the integrity of the work as a whole, and have given final approval for the version to be published.

Disclosures. Katie Linden, Conor McQuillan and Paul Brennan have nothing to disclose. Ian B. A. Menown has received grants to institution, honoraria and/or conference sponsorship from Biosensors, Boston Scientific, Meril Life, Orbus Neich, Astra Zeneca, Amgen, Bayer, Boehringer Ingelheim, Daichii Sankyo, Lilly, Bristol Myers Squibb, Pfizer, and Sanofi Aventis.

Compliance with Ethics Guidelines. This article is based on previously conducted studies and does not involve any new studies of human or animal subjects performed by any of the authors.

Open Access. This article is distributed under the terms of the Creative Commons Attribution-NonCommercial 4.0 International License (http://creativecommons.org/licenses/ by-nc/4.0/), which permits any noncommercial use, distribution, and reproduction in any 
medium, provided you give appropriate credit to the original author(s) and the source, provide a link to the Creative Commons license, and indicate if changes were made.

\section{REFERENCES}

1. Saito S, Krucoff MW, Nakamura S, et al. JapanUnited States of America Harmonized Assessment by Randomized Multicentre Study of OrbusNEich's Combo StEnt (Japan-USA HARMONEE) study: primary results of the pivotal registration study of combined endothelial progenitor cell capture and drug-eluting stent in patients with ischaemic coronary disease and non-ST-elevation acute coronary syndrome. Eur Heart J. 2018;39(26):2460-8.

2. Colombo A et al. One-year clinical outcomes of the bio-engineered COMBO stent: primary results from the 3614 all-comer patients in the COMBO Collaboration. Presented at American College of Cardiology Annual Scientific Sessions 2018. 11th March 2018; Orlando, FL.

3. Kufner S, Joner M, Thannheimer A, et al. Ten-year clinical outcomes from a trial of three limus-eluting stents with different polymer coatings in patients with coronary artery disease: results from the ISARTEST 4 randomized trial. Circulation. 2019;139;325-33.

4. El-Hayek G, Bangalore S, Casso Dominguez A, et al. Meta-analysis of randomized clinical trials comparing biodegradable polymer drug-eluting stent to second-generation durable polymer drug-eluting stents. JACC Cardiovasc Interv. 2017;10(5):462-73.

5. Urban P, Meredith IT, Abizaid A, et al. Polymer-free drug-coated coronary stents in patients at high bleeding risk. N Engl J Med. 2015;373:2038-47.

6. Lipiecki J, Brunel P, Morice MC, et al. Biolimus A9 polymer-free coated stents in high bleeding risk patients undergoing complex PCI: evidence from the LEADERS FREE randomised clinical trial. EuroIntervention. 2018;14(4):e418-25.

7. Rozemeijer R, Stein M, Voskuil M, et al. Randomized all-comers evaluation of a permanent polymer zotarolimus-eluting stent versus a polymer-free amphilimus-eluting stent:(ReCre8) a multicenter, non-inferiority trial. Circulation. 2018;139:67-77.

8. McQuillan C, Gray A, Kearney A, et al. Advances in clinical cardiology 2017: a summary of key clinical trials. Adv Ther. 2017;34(7):1503-27.
9. Aller D. TCT.18: Absorb stents are dead-but what about BVS in general? https://www.cardiovas cularbusiness.com/topics/coronary-interventionsurgery/absorb-stents-dead-what-about-bvs-general. Accessed 18 Feb 2019.

10. Neumann FJ, Sousa-Uva M, Ahlsson A, et al. 2018 ESC/EACTS guidelines on myocardial revascularization. Eur Heart J. 2019;40(2):87-165.

11. Byren RA. Intracoronary scaffold assessment a randomised evaluation of absorb in myocardial infarction (ISAR-Absorb MI) trial. Presented at European Society of Cardiology Congress 2018. 28th August 2018; Munich, Germany.

12. Stone GW, Ellis SG, Gori T, et al. Blinded outcomes and angina assessment of coronary bioresorbable scaffolds: 30-day and 1-year results from the ABSORB IV randomised trial. Lancet. 2018;392(10157):1530-40.

13. Smits PC. A bioresorbable everolimus-eluting scaffold versus a metallic everolimus-eluting stentCOMPARE-ABSORB. Presented at transcatheter cardiovascular therapeutics meeting 2018, 25th September 2018; San Diego, CA.

14. Garcia-Garcia HM, Haude M, Kuku K, et al. In vivo serial invasive imaging of the second-generation drug-eluting absorbable metal scaffold (MagmarisDREAMS 2G) in de novo coronary lesions: insights from the BIOSOLVE-II first-in-man trial. Int J Cardiol. 2018;255:22-8.

15. McMahon S, Bertollo NO, Cearbhaill ED, et al. Bioresorbable polymer stents: a review of material progress and prospects. Prog Polym Sci. 2018;83:79-96.

16. Waksman R, Serra A, Loh JP, et al. Drug-coated balloons for de novo coronary lesions: results from the Valentines II trial. EuroIntervention. 2013;5:613-9.

17. Tuomas $\mathrm{T}$, et al. Drug-eluting balloon for the treatment of de novo coronary artery lesions in patients with high bleeding risk-a randomized controlled single-blind multicenter trial (DEBUT). Presented at EuroPCR annual scientific sessions 2018. 23rd May 2018; Paris, France.

18. Jeger RV, Farah A, Ohlow MA, et al. Drug-coated balloons for small coronary artery disease (BASKETSMALL 2): an open-label randomised non-inferiority trial. Lancet. 2018;392(10150):849-56.

19. Walsh $S$ et al. The CONSISTENT CTO trial: CONventional antegrade vs Sub-Intimal Synergy sTENTing in chronic total occlusions. Presented at EuroPCR annual scientific sessions 2018. 24th May 2018; Paris, France. 
20. Xaplanteris P, Fournier S, Pijls NH, et al. Five-year outcomes with PCI guided by fractional flow reserve. N Engl J Med. 2018;379(3):250-9.

21. Al-Lamee R, Howard JP, Shun-Shin MJ, et al. Fractional flow reserve and instantaneous wave-free ratio as predictors of the placebo-controlled response to percutaneous coronary intervention in stable single-vessel coronary artery disease: physiology-stratified analysis of ORBITA. Circulation. 2018;138(17):1780-92.

22. Smits PC, Abdel-Wahab M, Neumann FJ, et al. Fractional flow reserve-guided multivessel angioplasty in myocardial infarction. $\mathrm{N}$ Engl J Med. 2017;376(13):1234-44.

23. Smits P. Two year results of COMPARE-ACUTE randomised trial of FFR-guided complete revascularization versus infarct artery only treatment in multivessel STEMI patients. Presented at EuroPCR Annual Scientific Sessions 2018. 23rd May 2018; Paris, France.

24. Thiele H, Akin I, Sandri M, et al. PCI strategies in patients with acute myocardial infarction and cardiogenic shock. $\mathrm{N}$ Engl J Med. 2017;377(25):2419-32.

25. Thiele H, Akin I, Sandri M, et al. One-year outcomes after PCI strategies in cardiogenic shock. N Engl J Med. 2018;379(18):1699-710.

26. Kofoed KF, Kelbæk H, Hansen PR, et al. Early versus standard care invasive examination and treatment of patients with non-st-segment elevation acute coronary syndrome: the VERDICT randomized controlled trial. Circulation. 2018;138:2741-50.

27. Rapid Assessment of Potential Ischaemic Heart Disease With CTCA (RAPID-CTCA). https:// clinicaltrials.gov/ct2/show/NCT02284191. Accessed 18 Feb 19.

28. Fearon WF. FAST-FFR (FFR angio $_{\text {accuracy versus }}$ standard FFR). Presented at transcatheter cardiovascular therapeutics meeting 2018, 24th September 2018; San Diego, CA.

29. Ford TJ, Stanley B, Good R, et al. Stratified medical therapy using invasive coronary function testing in angina: the CorMicA trial. J Am Coll Cardiol. 2018;72((23 Part A)):2841-55.

30. Valgimigli M, Frigoli E, Leonardi S, et al. Radial versus femoral access and bivalirudin versus unfractionated heparin in invasively managed patients with acute coronary syndrome (MATRIX): final 1-year results of a multicentre, randomised controlled trial. Lancet. 2018;392(10150):835-48.
31. Waksman R. Assessment of coronary near-infrared spectroscopy imaging to detect vulnerable plaques and vulnerable patients. Presented at TCT 2018. September 24, 2018. San Diego, CA.

32. Cribier A, Eltchaninoff $\mathrm{H}$, Bash A, et al. Percutaneous transcatheter implantation of an aortic valve prosthesis for calcific aortic stenosis-first human case description. Circulation. 2002;106(24):3006-8.

33. Baumgartner H, Falk V, Bax JJ, et al. 2017 ESC/ EACTS Guidelines for the management of valvular heart disease The Task Force for the Management of Valvular Heart Disease of the European Society of Cardiology (ESC) and the European Association for Cardio-Thoracic Surgery (EACTS). Eur Heart J. 2017;38(36):2739.

34. Leon MB, Smith CR, Mack MJ, et al. Transcatheter or surgical aortic-valve replacement in intermediate-risk patients. $\mathrm{N}$ Engl J Med. 2016;374(17):1609-20.

35. Reardon MJ, Van Mieghem NM, Pompa JJ, et al. Surgical or transcatheter aortic-valve replacement in intermediate-risk patients. $\mathrm{N}$ Engl J Med. 2017;376:1321-31.

36. Thyregod HGH, editor. Five-year outcomes from the all-comers nordic aortic valve intervention randomized clinical trial in patients with severe aortic valve stenosis. Presentation at American College of cardiology annual scientific sessions 2018; 2018 10th November 2018; Orlando, FL.

37. Waksman R, Rogers $\mathrm{T}$, Torguson $\mathrm{R}$, et al. Transcatheter aortic valve replacement in low-risk patients with symptomatic severe aortic stenosis. J Am Coll Cardiol. 2018;72(18):2095-105.

38. Chakravarty T, Sondergaard L, Friedman J, et al. Subclinical leaflet thrombosis in surgical and transcatheter bioprosthetic aortic valves: an observational study. Lancet. 2017;389(10087):2383-92.

39. Bekeredjian R, Szabo G, Balaban U, et al. Patients at low surgical risk as defined by the Society of Thoracic Surgeons Score undergoing isolated interventional or surgical aortic valve implantation: inhospital data and 1-year results from the German Aortic Valve Registry (GARY). Eur Heart J. 2018. https://doi.org/10.1093/eurheartj/ehy699.

40. Didier R, Eltchaninoff $H$, Donzeau-Gouge P, et al. 5 -year clinical outcame and valve durability after transcatheter aortic valve replacement in high-risk patients: the FRANCE-2 registry. Circulation. 2018. Presented at ESC Congress 2018. 29th August 2018. Munich, German.

41. Barbanti M, Petronio AS, Ettori F, et al. 5-year outcomes after transcatheter aortic valve implantation 
with CoreValve prosthesis. JACC Cardiovasc Interv. 2015;8(8):1084-91.

42. Eltchaninoff $\mathrm{H}$, editor. Clinical experiences and echo data from Rouen. Presented at transcatheter valve therapies. 17th June 2016; Chicago, IL.

43. Reichenspurner H, Schaefer A, Schafer U, et al. Selfexpanding transcatheter aortic valve system for symptomatic high-risk patients with severe aortic stenosis. J Am Coll Cardiol. 2017;70(25):3127-36.

44. Reichenspurner $\mathrm{H}$, editor One-year outcomes of CENTERA-EU trial assessing a novel self-expanding transcatheter heart valve. Presented at Euro PCR 2018, 22nd May 2818; Paris, France.

45. Sondergaard L, Rodes-Cabau J, Linke AH, et al. Oneyear outcomes with a self-expanding, repositonable transcatheter heart valve in severe aortic stenosis patients: PORTICO-I. J Am Coll Cardiol. 2018. https://doi.org/10.1016/j.jacc.2018.09.014.

46. Manoharan G, Van Mieghem NM, Windecker S. 1-year outcomes with the Evolut R self-expanding transcatheter aortic valve from the international FORWARD study. JACC Cardiovasc Interv. 2018;11(22):2326-34.

47. Thiele $\mathrm{H}$, editor. A $2 \times 2$ randomized trial of selfexpandable vs balloon-expandable valves and general vs local anesthesia in patients undergoing transcatheter aortic valve implantation. Presented at transcatheter cardiovascular therapeutics meeting 2018, 23rd September 2018; San Diego, CA.

48. Allen KB, Chhatriwalla AK, Saxon JT, et al. Bioprosthetic valve fracture: technical insights from a multicenter study. J Thorac Cardiovasc Surg. 2019. https://doi.org/10.1016/j.jtcvs.2019.01.073.

49. Worhle J. ADVERTORIAL: Sentinel Cerebral Protection System provides reassurance to both physicians and patients. https://cardiovascularnews.com/ advertorial-sentinel-cerebral-protection-system-pro vides-reassurance-to-both-physicians-and-patients/ 2018. Accessed 25 Nov 2018.

50. Schafer U. Safety and efficacy of protected cardiac intervention: clinical evidence for sentinel cerebral embolic protection. Interv Cardiol Rev. 2017;12(2):128-32.

51. Makker R. Presented at cardiovascular research technologies 2018. 3rd March 2018; Washington, DC.

52. Overtchouk P, Guedeney P, Rouanet S, et al. Longterm mortality and early valve dysfunction according to anticoagulation use: the FRANCE-TAVI registry. J Am Coll Cardiol. 2019;73(1):13-21.
53. Neale T. GALILEO trial of rivaroxaban after TAVR stopped early for harm. https://www.tctmd.com/ news/galileo-trial-rivaroxaban-after-tavr-stopped-ear ly-harm2018. Accessed 25 Nov 2018.

54. Barbanti M. PCR LV 2018: early discharge feasible for unselected TAVI patients https://cardiovas cularnews.com/pcr-lv-2018-early-discharge-feasiblefor-unselected-tavi-patients/2018. Accesssed 25 Nov 2018.

55. Feldman T, Foster E, Glower DG, et al. Percutaneous repair or surgery for mitral regurgitation. N Engl J Med. 2011;364(15):1395-406.

56. Obadia JF, Messika-Zeitoun D, Leurent G, et al. Percutaneous repair or medical treatment for secondary mitral regurgitation. $\mathrm{N}$ Engl $\mathrm{J}$ Med. 2108;379(24):2297-2306.

57. Stone G, Lindenfeld J, Abraham WT, et al. Transcatheter mitral-valve repair in patients with heart failure. N Engl J Med. 2018;379(24):2307-18.

58. Wood S. COAPT: MitraClip reduces repeat hospitalizations, mortality in functional MR patients with severe HF. https://www.tctmd.com/news/ coapt-mitraclip-reduces-repeat-hospitalizations-mor tality-functional-mr-patients-severe-hf2018. Accessed 25 Nov 2018.

59. RESHAPE-HF2 investigators and sponsors. A clinical evaluation of the safety and effectiveness of the MitraClip system in the treatment of clinically significant functional mitral regurgitation (ReshapeHF2). https://clinicaltrials.gov/ct2/show/NCT02444 338. Accessed 25 Nov 2018.

60. Latib A, editor. 6 month results from TRI-REPAIR. Presented at London Valve PCR 2018. 9th September 2018; London, England.

61. Iverson K, Ihlemann N, Gill SU, et al. Artial oral versus intravenous antibiotic treatment of endocarditis. N Engl J Med. 2019;380(5):415-24.

62. Erbel R, Aboyans V, Boileau C, et al. 2014 ESC Guidelines on the diagnosis and treatment of aortic diseases. Eur Heart J. 2014;35(41):2873-296.

63. Mullen M, editor. AIMS-Aortic Irbesartan Marfan Study. Presented at European Society of Cardiology Congress 2018. 28th August 2018; Munich, Germany.

64. Zinman B, Wanner C, Lachin JM, et al. Empagliflozin, cardiovascular outcomes, and mortality in type 2 diabetes. N Engl J Med. 2015;373:2117-28.

65. Kosiborod M, Lam CSP, Kohsaka S, et al. Cardiovascular events associated with SGLT-2 inhibitors 
versus other glucose-lowering drugs the CVD-REAL 2 study. J Am Coll Cardiol. 2018;71(23):2628-39.

66. Wiviott SD, Raz I, Bonaca MP, et al. Dapagliflozin and cardiovascular outcomes in type 2 diabetes. N Engl J Med. 2019;380(4):347-57.

67. Rådholm K, Figtree G, Perkovic V, et al. Canagliflozin and heart failure in type 2 diabetes mellitus: results from the CANVAS program. Circulation. 2018;138(5):458-68.

68. Kumbhani D. EMPA-HEART Cardiolink-6-EMPAHEART. Presented at American Heart Association Annual Scientific Sessions 2018. 11th November 2018; Chicago, IL.

69. Scirica BM, Bhatt DL, Braunwald E, et al. Saxagliptin and cardiovascular outcomes in patients with type 2 diabetes mellitus. N Engl J Med. 2013;369:1317-26.

70. White WB, Cannon CP, Heller SR, et al. Alogliptin after acute coronary syndrome in patients with type 2 diabetes. N Engl J Med. 2013;369:1327-35.

71. McMurray JJV, Ponikowski P, Bolli GB, et al. Effects of vildagliptin on ventricular function in patients with type 2 diabetes mellitus and heart failure: a randomized placebo-controlled trial. J Am Coll Cardiol Heart Fail. 2018;6(1):8-17.

72. Victor RG, Lynch K, Li N, et al. A cluster-randomized trial of blood-pressure reduction in black barbershops. N Engl J Med. 2018;378(14):1291-301.

73. Webster R, Salam A, Silva HA, et al. Fixed low-dose triple combination antihypertensive medication vs usual care for blood pressure control in patients with mild to moderate hypertension in Sri Lanka a randomized clinical trial. J Am Med Assoc. 2018;320(6):566-79.

74. Kandzari DE, Bohm M, Mahfoud F, et al. Effect of renal denervation on blood pressure in the presence of antihypertensive drugs: 6-month efficacy and safety results from the SPYRAL HTN-ON MED proofof-concept randomised trial. Lancet. 2018; 391(10137):2346-55.

75. Gupta A, Mackay J, Whitehouse A, et al. Long-term mortality after blood pressure-lowering and lipidlowering treatment in patients with hypertension in the Anglo-Scandinavian Cardiac Outcomes Trial (ASCOT) Legacy study: 16-year follow-up results of a randomised factorial trial. Lancet. 2018;392(10153): 1127-37.

76. Gaziano JM, Brotons C, Coppolecchia R, et al. Use of aspirin to reduce risk of initial vascular events in patients at moderate risk of cardiovascular disease (ARRIVE): a randomised, double-blind, placebocontrolled trial. Lancet. 2018;392(10152):1036-46.
77. Bowman L, Mafham M, Wallendszus K, et al. Effects of aspirin for primary prevention in persons with diabetes mellitus. N Engl J Med. 2018;379(16): 1529-39.

78. Sabatine MS, Giugliano RP, Keech AC, et al. Evolocumab and clinical outcomes in patients with cardiovascular disease. N Engl J Med. 2017;376: 1713-22.

79. Schwartz GG, Steg PG, Szarek M, et al. Alirocumab and cardiovascular outcomes after acute coronary syndrome. N Engl J Med. 2018;379(22):2097-2107.

80. Bowman L, Mafham M, Wallendszus K, et al. Effects of n-3 fatty acid supplements in diabetes mellitus. N Engl J Med. 2018;379(16):1540-50.

81. Yokoyama M, Origasa H, Matsuzaki M, et al. Effects of eicosapentaenoic acid on major coronary events in hypercholesterolaemic patients (JELIS): a randomised open-label, blinded endpoint analysis. Lancet. 2007;369:1090-8.

82. Bhatt DL, Steg PG, Miller M, et al. Cardiovascular risk reduction with icosapent ethyl for hypertriglyceridemia. N Engl J Med. 2019;380(1):11-22.

83. Bohula EA, Wiviott SD, McGuire DK, et al. Cardiovascular safety of lorcaserin in overweight or obese patients. N Engl J Med. 2018;379(12):1107-17.

84. Newby DE, Adamson PD, Berry C, et al. Coronary CT angiography and 5-year risk of myocardial infarction. N Engl J Med. 2018;379(10):924-33.

85. Ridker PM, Everett BM, Thuren T, et al. Antiinflammatory therapy with canakinumab for atherosclerotic disease. $\mathrm{N}$ Engl J Med. 2017;377(12):1119-31.

86. Ridker PM, Everett BM, Pradhan A, et al. Low-dose methotraxate for the prevention of atherosclerotic events. N Engl J Med. 2019;380(8):752-62.

87. Valgimigli M, Bueno H, Byrne RA, et al. 2017 ESC focused update on dual antiplatelet therapy in coronary artery disease developed in collaboration with EACTS. Eur J Cardiothorac Surg. 2017;53(1):34-78.

88. Hahn JY, Song YB, Oh JH, et al. 6-month versus 12-month or longer dual antiplatelet therapy after percutaneous coronary intervention in patients with acute coronary syndrome (SMART-DATE): a randomised, open-label, non-inferiority trial. Lancet. 2018;391(10127):1274-84.

89. Vranckx P, Valgimigli M, Jüni $P$, et al. Ticagrelor plus aspirin for 1 month, followed by ticagrelor monotherapy for 23 months vs aspirin plus clopidogrel or ticagrelor for 12 months, followed by 
aspirin monotherapy for 12 months after implantation of a drug-eluting stent: a multicentre, openlabel, randomised superiority trial. Lancet. 2018;392(10151):940-9.

90. Pasceri V, Patti G, Nusca A, et al. Randomized trial of atorvastatin for reduction of myocardial damage during coronary intervention: results from the ARMYDA (Atorvastatin for Reduction of Myocardial Damage During Angioplasty) study. Circulation. 2004;110(6):674-8.

91. Briguori C, Visconti G, Focaccio A, et al. Novel approaches for preventing or limiting events (Naples) II trial: impact of a single high loading dose of atorvastatin on periprocedural myocardial infarction. J Am Coll Cardiol. 2009;54(23):2157-63.

92. Berwanger O, Santucci EV, de Andrade Jesuíno I, et al. Effect of loading dose of atorvastatin prior to planned percutaneous coronary intervention on major adverse cardiovascular events in acute coronary syndrome: the SECURE-PCI randomized clinical trial. J Am Med Assoc. 2018;319(13):1331-40.

93. Shah AS, Anand A, Strachan FE, et al. High-sensitivity troponin in the evaluation of patients with suspected acute coronary syndrome: a steppedwedge, cluster-randomised controlled trial. Lancet. 2018;392(10151):919-28.

94. Dans AL, Connolly SJ, Wallentin L, et al. Concomitant use of antiplatelet therapy with dabigatran or warfarin in the randomized evaluation of long-term anticoagulation therapy (RE-LY) trial. Circulation. 2013;127:634-40.

95. Matsumura-Nakano Y, Shizuta S, Komasa A, et al. An open-label randomized trial comparing oral anticoagulation with and without single antiplatelet therapy in patients with atrial fibrillation and stable coronary artery disease beyond one year after coronary stent implantation: the OAC-ALONE study. Circulation. 2019;139:604-16.

96. Packer DL. Catheter ablation vs antiarrhythmic drug therapy in atrial fibrillation: the results of the CABANA multicenter international randomized clinical trial. Presented at Heart Rhythm Society 2018. 10th May 2018; Boston, MA.

97. Poole JE. Recurrence of atrial arrhythmias in the catheter ablation versus antiarrhythmic drug therapy for atrial fibrillation (CABANA) Trial. Presented at European Society of Cardiology Congress 2018. 26th August 2018; Munich, Germany.

98. Connolly S. Interim report on the ANNEXA-4 study: andexanet for reversal of anticoagulation in factor Xa associated acute major bleeding. Presented at American College of Cardiology Scientific Sessions 2018. 12th March 2018; Orlando, FL.
99. Ezekowitz MD, Pollack CV, Halperin JL, et al. Apixaban compared to heparin/vitamin $\mathrm{K}$ antagonist in patients with atrial fibrillation scheduled for cardioversion: the EMANATE trial. Eur Heart J. 2018;39(32):2959-71.

100. Priori SG, Blomstrom-Lundqvist C, Mazzanti A, et al. 2015 ESC Guidelines for the management of patients with ventricular arrhythmias and the prevention of sudden cardiac death: the task force for the management of patients with ventricular arrhythmias and the prevention of sudden cardiac death of the European Society of Cardiology (ESC). Eur Heart J. 2015;36(41):2793-867.

101. Al-Khatib SM, Stevenson WG, Ackerman MJ, et al. 2017 AHA/ACC/HRS guideline for management of patients with ventricular arrhythmias and the prevention of sudden cardiac death. Circulation. 2017;138(13):272-391.

102. Olgin JE, Pletcher MJ, Vittinghoff E, et al. Wearable cardioverter-defibrillator after myocardial infarction. N Engl J Med. 2018;379(13):1205-15.

103. Zei PC, Mak R. Noninvasive stereotactic radioablation for ventricular tachycardia ENCORE-VT (EPGuided Noninvasive Cardiac Radioablation): is the sequel as good as the original? Circulation. $2019 ; 139 ; 322-4$.

104. Abdelrahman M, Subzposh FA, Beer D, et al. Clinical outcomes of his bundle pacing compared to right ventricular pacing. J Am Coll Cardiol. 2018;71(20):2319-30.

105. McMurray JJ, Packer M, Desai AS, et al. Angiotensinneprilysin inhibition versus enalapril in heart failure. N Engl J Med. 2014;371(11):993-1004.

106. Velazquez EJ, Morrow DA, DeVore AD, et al. Angiotensin-neprilysin inhibition in acute decompensated heart failure. $\mathrm{N}$ Engl $\mathrm{J}$ Med. 2019;380(6):539-48.

107. Givertz MM, Stevenson LW, Costanzo MR, et al. Pulmonary artery pressure-guided management of patients with heart failure and reduced ejection fraction. J Am Coll Cardiol. 2017;70(15):1875-86.

108. Abraham J. CardioMEMS. Presented at American College of Cardiology Annual Scientific Sessions 2018. 12th March 2018; Orlando, FL.

109. Guide HF authors and sponsors. HemodynamicGUIDEd Management of Heart Failure (GUIDE-HF). https://clinicaltrials.gov/ct2/show/NCT03387813. Accessed 26 Nov 2018.

110. Koehler F, Koehler K, Deckwart O, et al. Efficacy of telemedical interventional management in patients with heart failure (TIM-HF2): a randomised, 
controlled, parallel-group, unmasked trial. Lancet. 2018;392(10152):1047-57.

111. Mehra MR, Goldstein DJ, Uriel N, et al. Two-year outcomes with a magnetically levitated cardiac pump in heart failure. $\mathrm{N}$ Engl $\mathrm{J}$ Med. 2018;378(15):1386-95.

112. Cardinale D, Colombo A, Lamantia G, et al. Anthracycline-induced cardiomyopathy: clinical relevance and response to pharmacologic therapy. J Am Coll Cardiol. 2010;55:213-20.

113. Cardinale D, Colombo A, Bacchiani G, et al. Early detection of anthracycline cardiotoxicity and improvement with heart failure therapy. Circulation. 2015;131:1981-8.
114. Avila MS, Ayub-Ferreira SM, de Barros Wanderley MR Jr, et al. Carvedilol for prevention of chemotherapy-related cardiotoxicity: the CECCY trial. J Am Coll Cardiol. 2018;71(20):2281-90.

115. Zannad F, Anker SD, Byra WM, et al. Rivaroxaban in patients with heart failure, sinus rhythm, and coronary disease. N Engl J Med. 2018;379(14): 1332-42.

116. Maurer MS, Schwartz JH, Gundapaneni B, et al. Tafamidis treatment for patients with transthyretin amyloid cardiomyopathy. $\mathrm{N}$ Engl J Med. 2018;379(11):1007-16. 